\title{
1 Complementary roles for parvalbumin and somatostatin interneurons in the generation of hippocampal gamma 3 oscillations
}

Pantelis Antonoudiou ${ }^{1}$, Yu Lin Tan ${ }^{1}$, Georgina Kontou ${ }^{3}$, A. Louise Upton ${ }^{1,2}$ and Edward O. Mann ${ }^{1,2}$

${ }^{1}$ Department of Physiology, Anatomy and Genetics, University of Oxford, Oxford, OX1 3PT, UK

${ }^{2}$ Oxford Ion Channel Initiative, University of Oxford, OX1 3PT, Oxford, UK

${ }^{3}$ Neuroscience, Physiology and Pharmacology, University College London

\section{Abstract}

Gamma-frequency oscillations $(30-120 \mathrm{~Hz})$ can be separated into fast $(>60 \mathrm{~Hz})$ and slow oscillations, with different roles in neuronal encoding and information transfer. While synaptic inhibition is important for synchronization across the gamma-frequency range, the role of distinct interneuronal subtypes in fast and slow gamma states remains unclear. Here, we used optogenetics to examine the involvement of parvalbumin ( $\mathrm{PV}+)$ and somatostatin $(\mathrm{SST}+)$ expressing interneurons in gamma oscillations in the mouse hippocampal CA3 ex vivo. Disrupting either PV+ or STT+ interneuron activity, via either photo-inhibition or photo-excitation, led to a decrease in the power of cholinergically-induced slow gamma oscillations. Furthermore, photo-excitation of SST+ interneurons induced fast gamma oscillations, which depended on both synaptic excitation and inhibition. Our findings support a critical role for both PV+ and SST+ interneurons in slow hippocampal gamma oscillations, and further suggest that STT+ interneurons are capable of switching the network between slow and fast gamma states. 


\section{Introduction}

Gamma oscillations ( $30-120 \mathrm{~Hz}$ ) are a common feature of active cortical networks, which have been proposed to contribute to local gain control (Sohal et al., 2009; Cardin et al., 2009; Sohal, 2016) and facilitate transmission between synchronised neuronal assemblies (Fries, 2005; Akam \& Kullmann, 2010; Fries, 2015). While the function of gamma oscillations remains debated (Burns, Xing \& Shapley, 2011; Butler \& Paulsen, 2014; Bastos, Vezoli \& Fries, 2015; Ray \& Maunsell, 2015; Womelsdorf \& Everling, 2015; Lasztóczi \& Klausberger, 2016; Sohal, 2016), changes in these rhythms continue to act as a useful marker of function and dysfunction in cortical circuit operations (Bragin et al., 1995; Fries et al., 2001; Herrmann \& Demiralp, 2005; Uhlhaas \& Singer, 2006; Basar-Eroglu et al., 2007; Uhlhaas \& Singer, 2010; Yamamoto et al., 2014; Spellman et al., 2015). There is a general consensus that the generation of gamma rhythms depends upon the spiking of inhibitory interneurons, which synchronise the firing of excitatory pyramidal cells via fast synaptic inhibition (Whittington, Traub \& Jefferys, 1995; Penttonen et al., 1998a; Csicsvari et al., 2003; Hajos, 2004; Mann et al., 2005; Hasenstaub et al., 2005; Bartos, Vida \& Jonas, 2007; Buzsáki \& Wang, 2012; Kim et al., 2016; Chen et al., 2017; Veit et al., 2017). Specifically, parvalbumin-expressing (PV+) interneurons, which target the perisomatic domain of pyramidal neurons, are thought to play the key role in generating and maintaining gamma oscillations in the brain (Csicsvari et al., 2003; Hajos, 2004; Mann et al., 2005; Gloveli et al., 2005; Hájos \& Paulsen, 2009; Tukker et al., 2013; Cardin, 2016; Penttonen et al., 1998b). PV+ interneurons are adapted for fast synchronisation of network activity, as they resonate at gamma frequencies and exert strong perisomatic inhibition that is capable of precisely controlling spike timing (Pike et al., 2000; Pouille \& Scanziani, 2001; Cardin et al., 2009; Bartos \& Elgueta, 2012; Hu, Gan \& Jonas, 2014; Kohus et al., 2016). Moreover, at least in the CA3 hippocampal subfield, the gamma oscillations recorded in the local field potential appear 
50 to directly reflect rhythmic perisomatic inhibitory currents (Mann et al., 2005; Oren, Hájos \&

51 Paulsen, 2010).

Recently, a selective role for PV+ interneurons in gamma-frequency synchronisation has been challenged by several studies performed in the primary visual cortex (Chen et al., 2017; Veit et al., 2017; Hakim, Shamardani \& Adesnik, 2018). In this brain region, it was shown that dendritetargeting somatostatin-expressing (SST+) interneurons were the main contributors for the generation of slow gamma oscillations, while $\mathrm{PV}+$ interneurons were more important for higher frequency synchronisation (Chen et al., 2017). Previous studies have found analogous roles for SST+ and PV+ interneurons in low- and high-frequency network synchronisation (Beierlein, Gibson \& Connors, 2000; Gloveli et al., 2005; Tukker et al., 2007; Craig \& McBain, 2015). However, it is not yet clear if it is the frequency tuning of each interneuronal circuit that varies across brain areas, or whether SST+ interneurons might play a more generic role in the generation of slow gamma oscillations.

The hippocampus displays both slow and fast gamma rhythms during theta activity, with slow gamma generated in CA3 and fast gamma propagated from entorhinal cortex (Bragin et al., 1995; Colgin et al., 2009; Schomburg et al., 2014; Lasztóczi \& Klausberger, 2016). The circuitry for slow gamma oscillations is preserved in hippocampal slices (Fisahn et al., 1998), and these models have been used extensively to show that PV+ interneurons are strongly phase-coupled to gamma oscillations, and contribute to rhythmogenesis (Hajos, 2004; Mann et al., 2005; Gloveli et al., 2005; Gulyás et al., 2010). However, the majority of interneurons are phase-coupled to ongoing slow gamma oscillations (Hajos, 2004; Gloveli et al., 2005; Oren et al., 2006), and it may be that SST+ interneurons play an important role in synchronising PV+ networks. Indeed, whether specific classes of CA3 interneuron are necessary and sufficient for the generation of slow gamma oscillations has not yet been tested. Here, we took advantage of optogenetic techniques (Nagel et al., 2003; Chow 
et al., 2010; Boyden et al., 2005) to test the involvement of PV+ and SST+ interneurons in cholinergically-induced gamma oscillations in the CA3 of acute hippocampal slices.

\section{Results}

\section{PV+ interneuron activity is necessary for cholinergically-induced gamma oscillations}

\section{in hippocampal CA3}

In order to test if the activity of PV+ interneurons is necessary for the generation of slow hippocampal gamma oscillations, we took advantage of optogenetic photo-inhibition (Chow et al., 2010). We injected PV-cre mice with AAV carrying the inhibitory proton pump archaerhodopsin (Arch3-eYFP or ArchT-GFP). Expression of Arch in PV-cre mice was restricted to the pyramidal cell layer indicating selective expression in perisomatic targeting PV+ interneurons (Fig. 1a) (Somogyi \& Klausberger, 2005; Royer et al., 2012; Hu, Gan \& Jonas, 2014). Intracellular recordings performed in opsin expressing cells demonstrated that these cells were fast-spiking and that sustained light illumination was able to produce robust hyperpolarisation, indicating functional expression of Arch in PV+ interneurons (Supplementary Fig. 1d-e).

Gamma oscillations were induced in hippocampal slices from PV-Arch mice in area CA3 using bath application of the cholinergic agonist carbachol (Cch - $5 \mu \mathrm{M})$. Local field potential recordings from the CA3 pyramidal cell layer revealed robust gamma oscillations that were centred around $30-40$ $\mathrm{Hz}$ with clear side peaks in the autocorrelogram (Supplementary Fig. 1a-c), as has been reported previously (Fisahn et al., 1998; Hajos, 2004; Mann et al., 2005). Overall, sustained photo-inhibition of PV+ interneurons using LED illumination $(<5 \mathrm{~mW})$ significantly decreased gamma power area $(0.82+/-0.068$ of baseline period, $t=2.59, p=0.029$, one sample t-test; Fig. $1 \mathrm{~b}-\mathrm{d})$, although increases in power were observed in some slices (Fig. 1d). A significant suppression was also 
observed in the period of $0.5-1.5$ seconds following light illumination termination $(0.85+/-0.022$ of baseline period, $t=6.70, p<0.001$, one sample t-test; Fig. $1 d)$. However, the light-induced changes in gamma power were reversible, as there were no significant changes in the gamma power area recorded during the baseline periods across trials $(F(4,116)=0.68, p=0.61$, rmANOVA $)$. The changes in gamma power were not accompanied by a consistent change in gamma frequency $(F(1.44,43.23)=1.25, p=0.288$, rmANOVA; Fig. $1 e)$, although there was a significant correlation between the changes in frequency and power area $(t=2.77, p=0.01$, Pearson correlation, Fig. 1f), suggesting a consistent disturbance to endogenous oscillatory activity.

While LED photo-inhibition of PV+ interneurons significantly modulated gamma power, the oscillations did not collapse. Pyramidal neurons make strong recurrent connections with PV+ interneurons (Mann, Radcliffe \& Paulsen, 2005; Oren et al., 2006; Hofer et al., 2011; Packer \& Yuste, 2011; Bartos \& Elgueta, 2012; Kohus et al., 2016), and it might be hard to break these feedback loops with photo-induced inhibitory currents. To test this possibility, we used long-lasting laser illumination with the prospect of biochemically silencing PV+ interneurons, by preventing synaptic release via terminal alkalisation (El-Gaby et al., 2016). PV+ interneurons expressing ArchT-GFP were illuminated with sustained green laser light (532 nm, approx. $18 \mathrm{~mW}$ for 20 seconds). Similar to the LED experiments, there were inconsistent network responses to PV+ interneuron photo-inhibition at the beginning of laser illumination $(1.00+/-0.087$ of baseline period, $t=0.04, p=1.00$, one sample t-test; Fig. 1g\&h). However, the power of the oscillation consistently decreased during sustained laser illumination $(0.57+/-0.086$ of baseline period, $t=5.00, p<0.001$, one sample t-test; Fig. 1g\&h) and remained suppressed in the light-off period following laser stimulation $(0.78+/-0.071$ of baseline period, $t=3.17, p=0.022$, one sample t-test; Fig. $1 \mathrm{~g}, \mathrm{~h}$ ). There was no consistent effect on the frequency of the oscillations $(F(1.92,23.08)=7.77, p=0.003$, rmANOVA; Fig. 1g\&i). Laser illumination of PV+ interneurons expressing only control fluorophore did not alter gamma oscillation power nor frequency (Supplementary Fig. 1g-i). This slow and selective process of decreasing 
122 gamma power is consistent with biochemical silencing of synaptic terminals (El-Gaby et al., 2016).

123 These results further support the importance of PV+ interneuron activity in generating gamma

124 oscillations in hippocampal area CA3 (Hajos, 2004; Mann et al., 2005; Gulyás et al., 2010; Tukker et

125 al., 2013). Residual gamma oscillations following photo-inhibition of PV+ interneurons may reflect

126 incomplete transfection of the PV+ network or the presence of a distinct oscillatory circuit. 


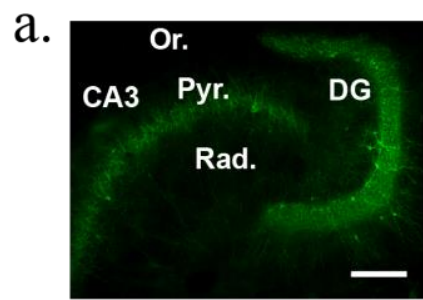

c.

Frequency $(\mathrm{Hz})$

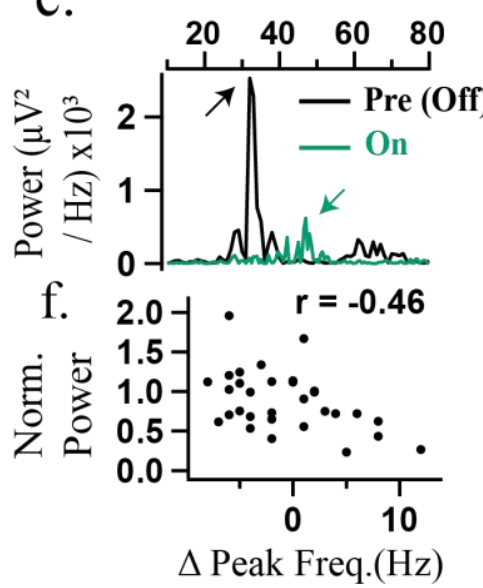

g.

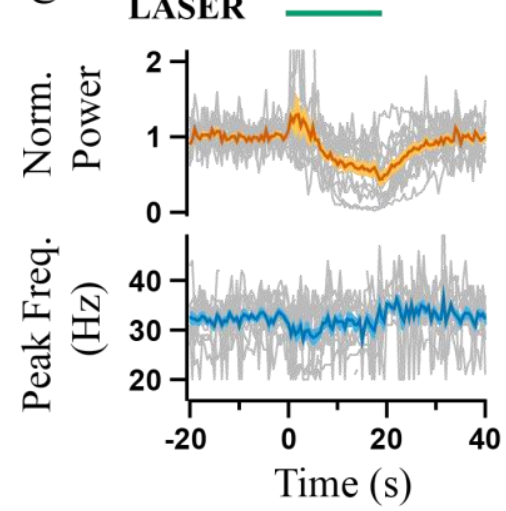

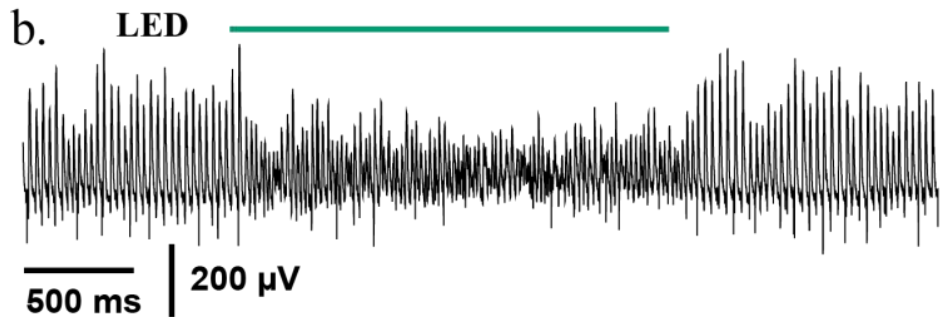

d.

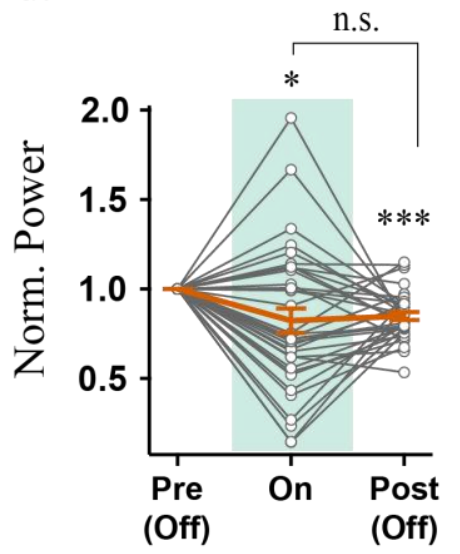

e.

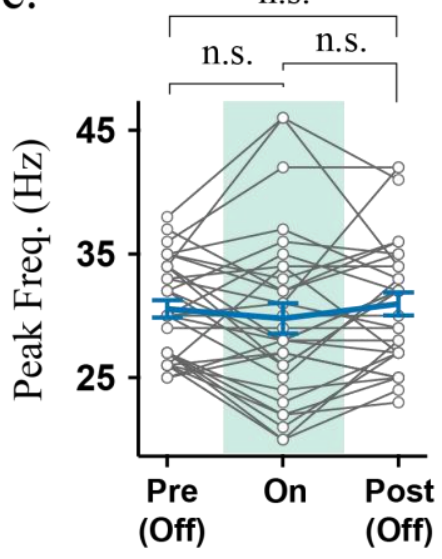

h.

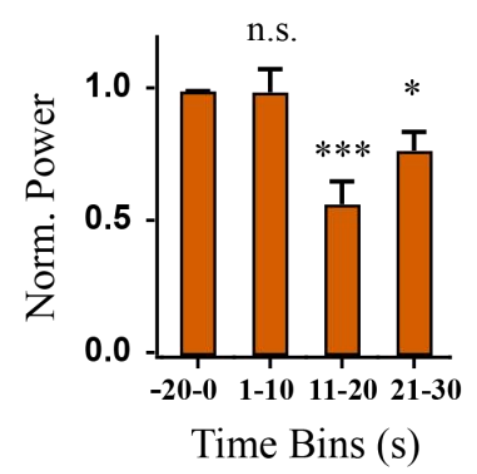

i.

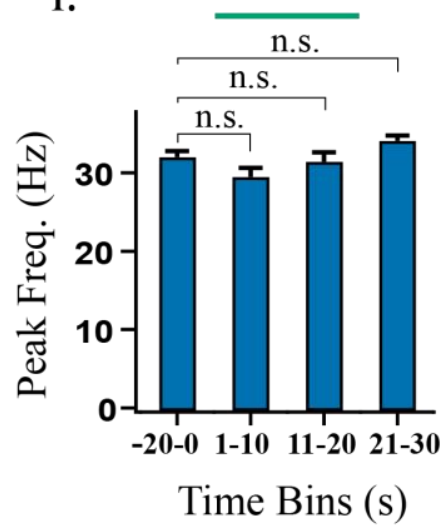

Figure 1: Sustained photo-inhibition of PV+ interneurons suppresses the power of gamma oscillations. ae) photo-inhibition with LED and g-i) photo-inhibition with laser experiments. a) Confocal image of ventral hippocampus slice from a PV-cre mouse injected intrahippocampally with AAV-Arch3 eYFP. CA3 = Cornu Ammonis 3, DG = Dentate Gyrus, Pyr. = stratum Pyramidale, Rad. = stratum Radiatum, Or. = Stratum Oriens. Scale bar $=200 \mu \mathrm{m}$. b) LFP recording from CA3 stratum pyramidale illustrating effect of $\mathrm{PV}+$ interneuron photo-inhibition (LED, $530 \mathrm{~nm}$, approx. $4.25 \mathrm{~mW}$ ) on gamma oscillations induced by the application of $5 \mu \mathrm{M}$ Cch. c) Representative power spectra before (black) and during (green) LED illumination (arrows indicate power spectrum peaks). d) Power area in the $20-100 \mathrm{~Hz}$ band normalised to baseline (Pre (Off)) during (On) and after LED stimulation (Off (Post)) $(\mathrm{n}=35)$. e) Peak frequency for experiments when the oscillation was not abolished $(n=31 / 35)$. f) Power area change versus peak frequency difference recorded between stimulation and baseline periods. g) Stronger photoinhibition was achieved using high power laser illumination (approx. $18.6 \mathrm{~mW}$ ). Top: Change in power area normalised to baseline. Bottom: Peak frequency of the oscillation calculated in 1 second bins across experiments ( $\mathrm{n}=$ 14). h) Mean change in power area normalised to baseline $(n=14)$. i) Mean peak frequency for trials when the oscillation was not abolished $(\mathrm{n}=13) .{ }^{*} \mathrm{p}<0.05$, ${ }^{*} \mathrm{p}<0.01$, ${ }^{* * *} \mathrm{p}<0.001, \mathrm{n} . \mathrm{s} . \mathrm{p}>=0.05$. Changes in peak frequency were analysed using rmANOVA, followed by post-hoc paired t-tests with correction for multiple comparisons. Solid brackets represent paired t-tests and standalone star symbols represent onesample t-test versus normalised baseline. Grey lines represent single experiments. Error bars and shaded area are SEM and coloured line the population mean. 


\section{Supplementary Figure 1 (Supporting Figure 1)}
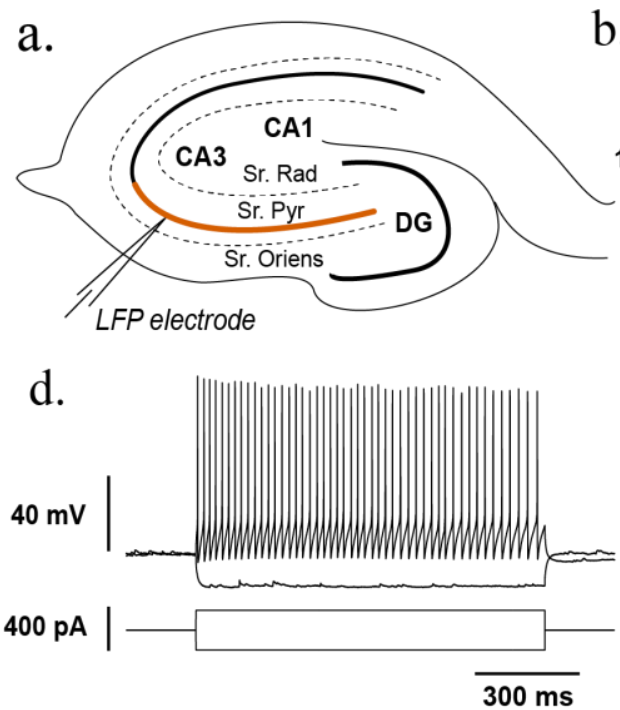

g.

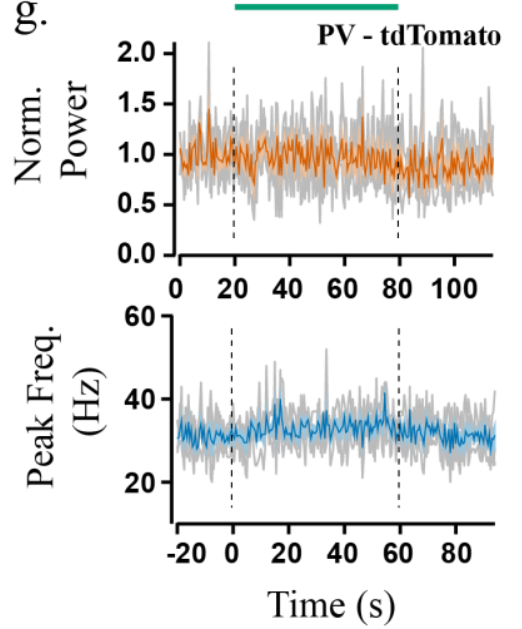

b.

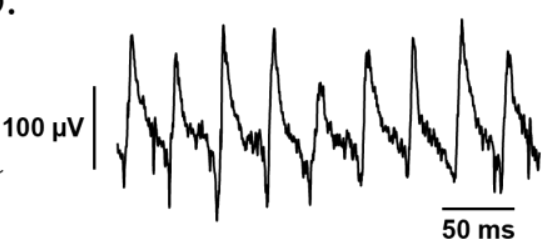

e.
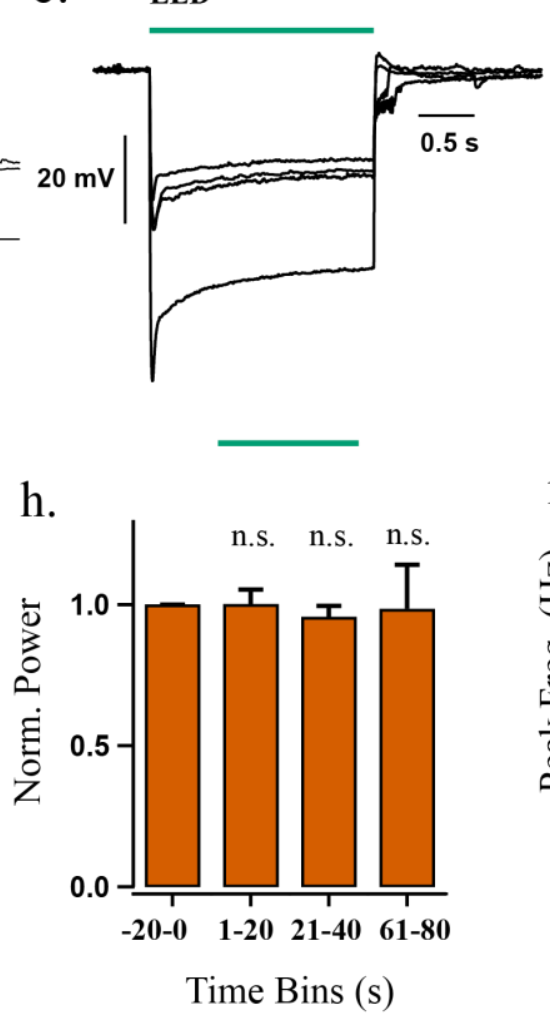

c.
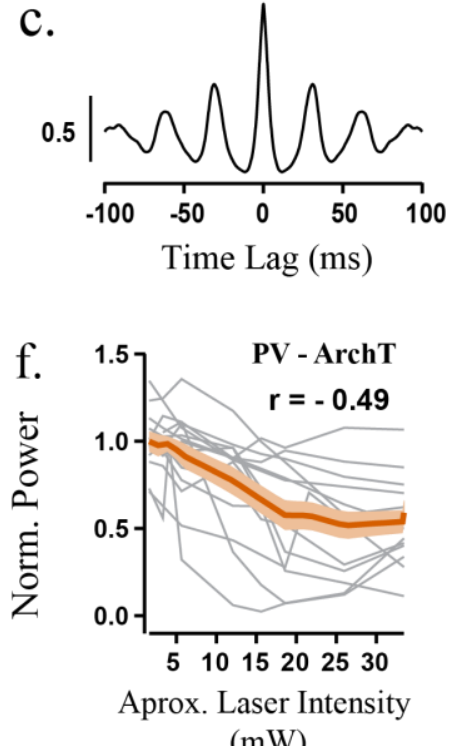

$(\mathrm{mW})$

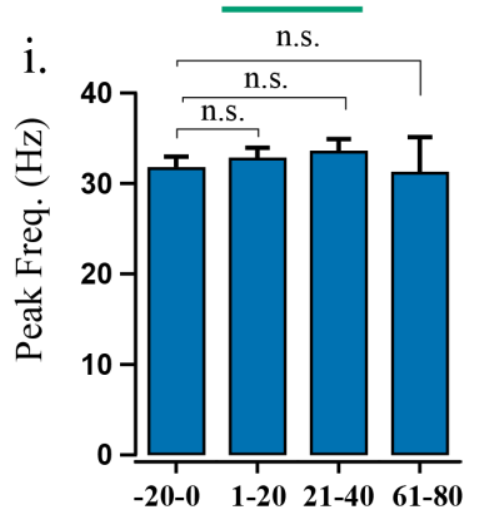

Time Bins (s)

Supplementary Figure 1: a-c) Recording gamma oscillations in hippocampal CA3. a) Illustration of the electrophysiological setup used to record gamma oscillations using a glass field electrode in stratum pyramidale of hippocampal area CA3 (coloured line indicates the CA3 area where recordings were obtained). b) Representative LFP recording from the ventral CA3 area obtained by the application of $5 \mu \mathrm{M}$ Cch. c) Autocorrelogram of the recording in b) illustrating that the oscillation is rhythmic with a period of $31 \mathrm{~ms}$. d-e) Validation of functional Arch expression in PV+ neurons. d) Current clamp recording of an ArchT-GFP expressing PV+ cell from CA3 area in response to steps of depolarising and hyperpolarising current injections. e) Potent hyperpolarisation of four PV+ interneurons during green light illumination in aCSF $(1.45 \mathrm{~mW})$. f) Power change between the last half of laser stimulation from baseline against approximate light intensity. Grey lines represent single experiments $(\mathrm{n}=14)$. The orange line is the average response and the orange shaded area represents SEM. g-i) Responses to laser illumination in control slices from PV-Ai9 mice. g) Top: Change in power-area normalised to baseline calculated in 1 second bins across experiments $(n=4)$. Bottom: Peak frequency of the oscillation calculated in 1 second bins across experiments $(n=4)$. Time between dotted lines indicates the duration of laser illumination (duration of approx. 1 minute and a light intensity of approx. 25-41 $\mathrm{mW}$ ). h-i) quantification of normalised power and peak frequency. n.s. $p>=0.05$. Changes in peak frequency were analysed using rmANOVA, followed by post-hoc paired t-tests with correction for multiple comparisons. Solid brackets represent paired t-tests and standalone star symbols represent one-sample t-test versus normalised baseline. Error bars and shaded area are SEM and coloured line the population mean. 


\section{hippocampal area ca3}

131

To examine if SST+ interneuron activity is also required during Cch-induced gamma oscillations in CA3, we injected the AAV-Arch vector (Arch3-eYFP or ArchT-GFP) intrahippocampally in SST-cre mice. Expression of Arch was restricted to the strata oriens, radiatum and lacunosum moleculare (Fig. 2a), suggesting expression in SST+ dendrite-targeting interneurons (Ma et al., 2006; LovettBarron et al., 2012; Muller \& Remy, 2014; Urban-Ciecko \& Barth, 2016). Whole-cell recordings were performed in opsin positive cells and indicated functional expression of Arch ( $n=4$, Supplementary Fig. 2a-b).

Unlike the experiments with PV+ photo-inhibition, sustained photo-inhibition of SST+ interneurons using LED illumination $(<5 \mathrm{~mW})$ reliably decreased gamma oscillation power $(0.69+/-0.057$ of baseline period, $t=5.40, p<0.001$, one sample t-test; Fig. $2 c, d$ ), which remained suppressed in the immediate period following SST + interneuron photoinhibition $(0.76+/-0.039$ of baseline period, $\mathrm{t}=$ $-6.26745, p<0.001$, one sample t-test; Fig. 1d). This post-light suppression was reversed from trial to trial $(F(4,164)=2046, p=0.048$, rmANOVA; all paired t-tests $t>2.81, p>0.07)$. In addition, light stimulation significantly modulated oscillation frequency $(F(1.25,39.97)=22.60, p<0.001$, rmANOVA), with an increase in frequency from $37.79+/-1.083 \mathrm{~Hz}$ to $43.00+/-1.466 \mathrm{~Hz}$ during light stimulation ( $t=4.74, p<0.01$, paired t-test), which reversed following light offset (Fig. $2 e$ ).

Stronger laser illumination in the first half of the stimulation period $(532 \mathrm{~nm}$, approx. $18 \mathrm{~mW}$ for 20 s) had similar effects as the LED experiment. Specifically, the power of Cch gamma oscillations decreased $(0.70+/-0.064$ of baseline, $t=4.76, p<0.01$, one-sample t-test $)$, and the peak frequency increased $(34.22+/-1.191 \mathrm{~Hz}$ to $38.60+/-1.868 \mathrm{~Hz}, \mathrm{t}=3.93, \mathrm{p}=0.017$, paired t-test; rmANOVA, $F(1.36,13.63)=5.47, p=0.027 ;$ Fig. $2 g-i)$. During the second half of the stimulation period, gamma power was strongly suppressed $(0.35+/-0.090$ of baseline, $t=7.23, p<0.01$, one sample $t$-test; Fig. 
$1532 \mathrm{~g}-\mathrm{h})$, often resulting in oscillation collapse (7/13 slices). This could indicate that silencing SST+

154 interneurons is sufficient to disrupt the hippocampal network during gamma oscillations and that

155 SST+ interneuron activity is necessary for proper maintenance of Cch-induced oscillations in the CA3

156 area of the hippocampus. Moreover, the frequency of the Cch-gamma oscillations remains

157 upregulated for the whole duration of laser illumination when the oscillations do not collapse (Fig.

$1582 \mathrm{i}$ vs Supplementary Fig.2d), but in each case remained below $60 \mathrm{~Hz}$, suggesting that SST+

159 interneurons can exert strong control over the frequency of slow gamma oscillations. 
a.

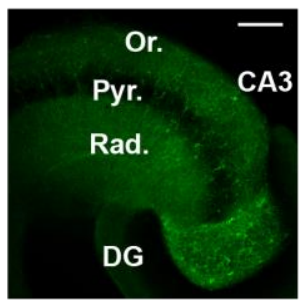

Frequency $(\mathrm{Hz})$

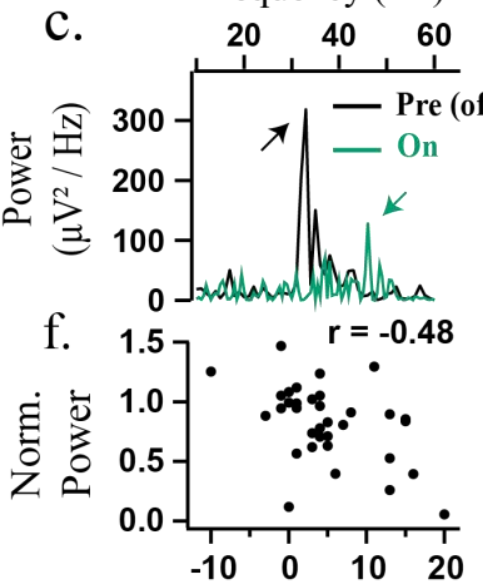

$\Delta$ Peak Freq. $(\mathrm{Hz})$

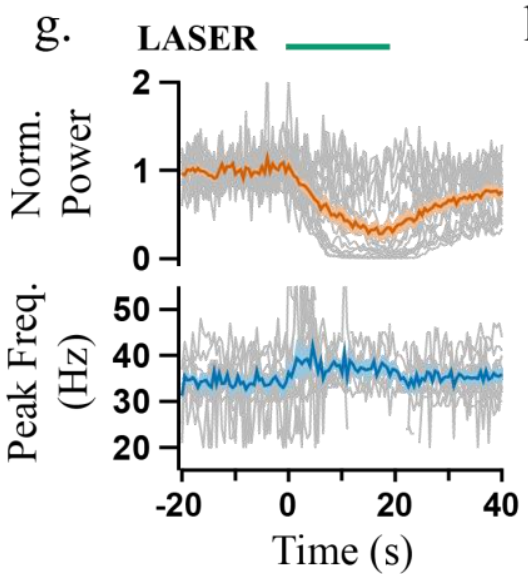

b.

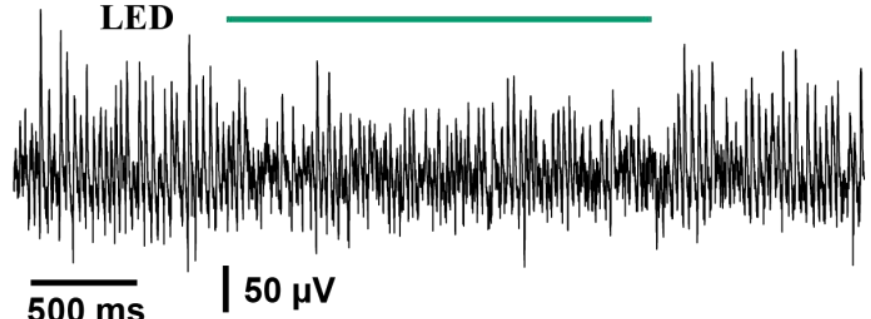

d.
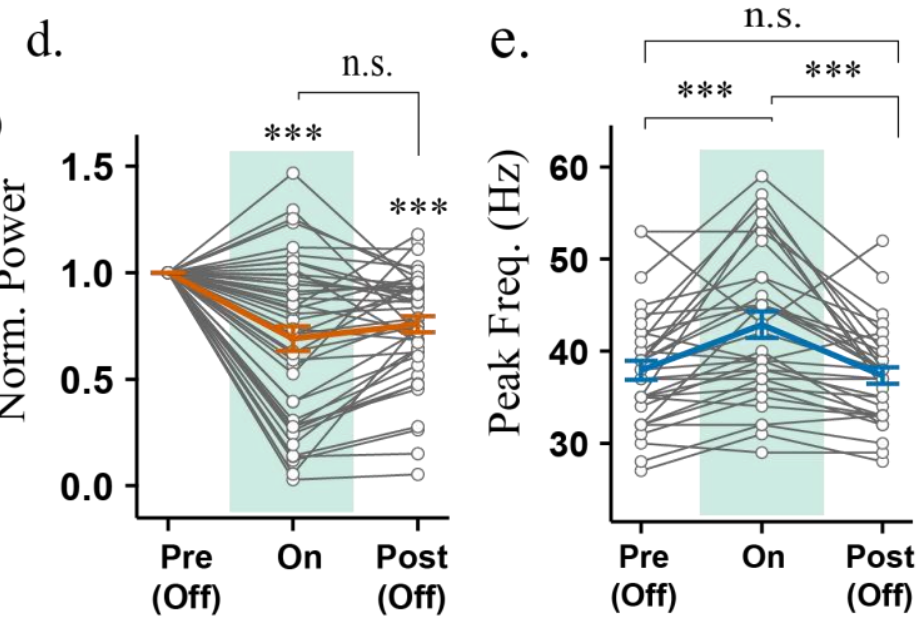

h.

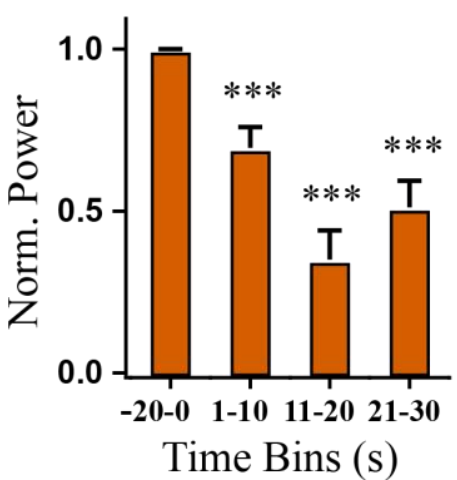

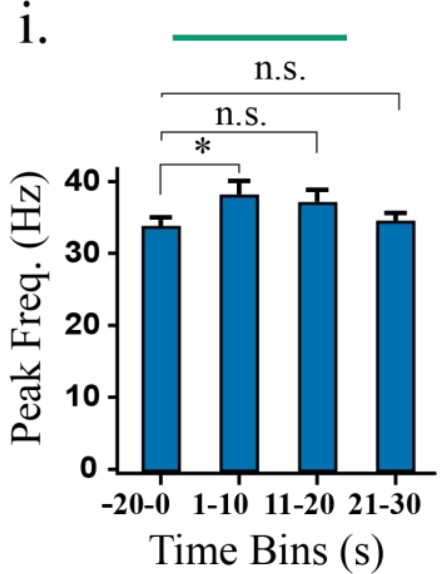

Figure 2: Sustained photo-inhibition of SST+ interneurons suppresses gamma power and increases frequency. a) Confocal image of ventral hippocampus slice from SST-cre mice with eYFP-Arch3 expression. $\mathrm{CA} 3$ = Cornu Ammonis 3, DG = Dentate Gyrus, Pyr. = stratum Pyramidale, Rad. = stratum Radiatum, Or. $=$ Stratum Oriens. Scale $b a r=200 \mu \mathrm{m}$. b) Representative LFP recordings from CA3 area illustrating effect of SST+ interneuron photo-inhibition (LED, $530 \mathrm{~nm}$, approx. $4.25 \mathrm{~mW}$ ) on gamma oscillations along with c) its respective power spectrum (arrows indicate power spectrum peaks). d) Power area in the $20-100 \mathrm{~Hz}$ band normalised to baseline (Pre (Off)) during (On) and after LED stimulation (Post $(\mathrm{Off}))(\mathrm{n}=44)$. e) Peak frequency for experiments when the oscillation was not abolished $(n=33 / 44)$. f) Power area change against peak frequency difference between stimulation and baseline periods. g) Top: Change in power-area normalised to baseline and Bottom: Peak frequency of the oscillation calculated in 1 second bins across experiments with high-power laser stimulation (approx. $18.6 \mathrm{~mW} ; \mathrm{n}=17) . \mathrm{h})$ Average power change normalised to baseline $(\mathrm{n}=17)$. i) Average peak frequency $(\mathrm{n}=11 / 17) . * \mathrm{p}<0.05, * * \mathrm{p}<0.01, * * * \mathrm{p}<0.001$, n.s. $\mathrm{p}>=0.05$. Changes in peak frequency were analysed using rmANOVA, followed by post-hoc paired t-tests with correction for multiple comparisons. Solid brackets represent paired t-tests and standalone star symbols represent one-sample $\mathrm{t}$-test versus normalised baseline. Grey lines represent single experiments, error bars and shaded area are SEM and coloured line the population average. 


\section{Supplementary Figure 2 (Supporting Figure 2)}
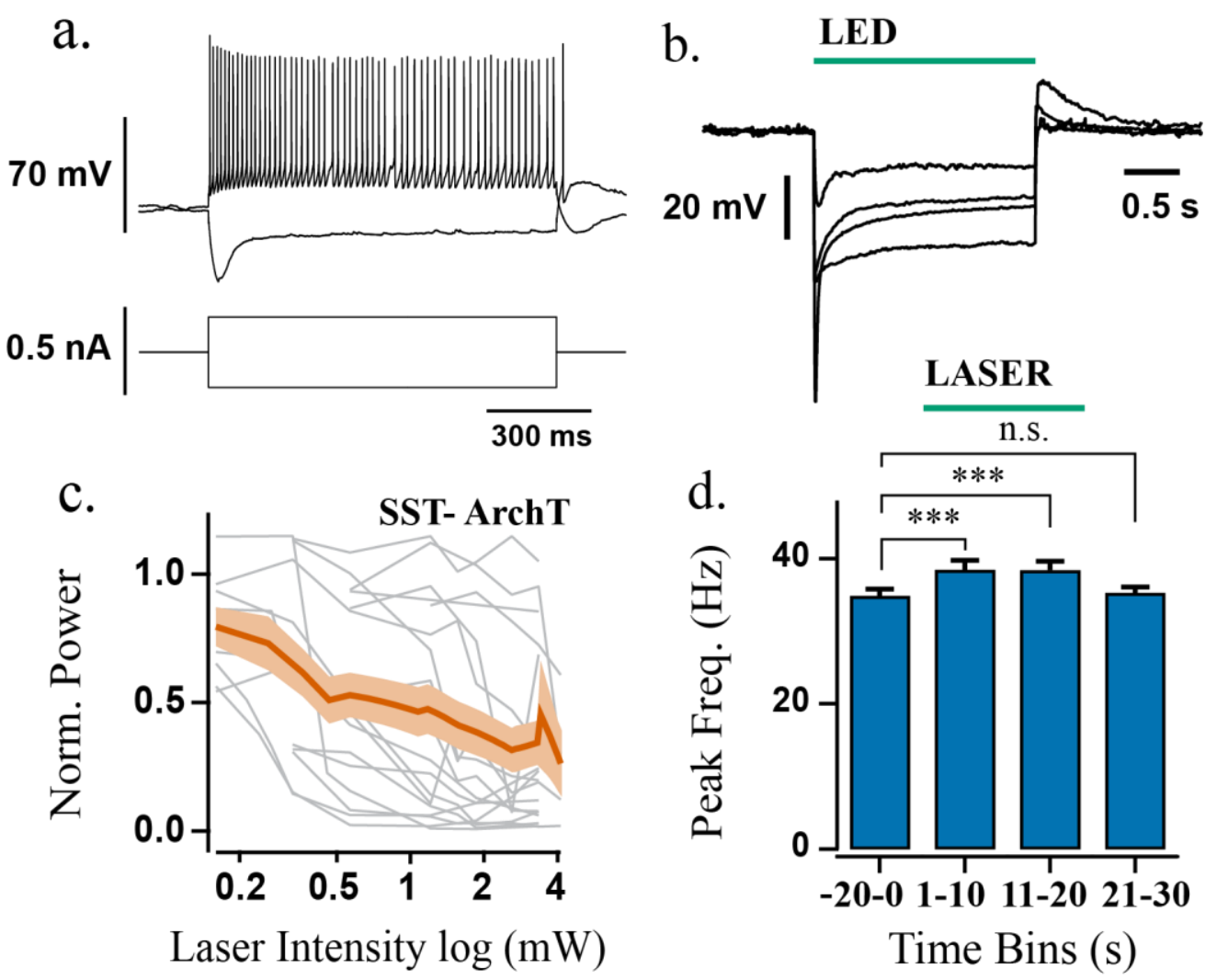

Supplementary Figure 2: a-b) Validation of functional opsin expression in SST+ interneurons. a) Current clamp recording of an SST+ cell from CA3 area in response to steps of depolarising and hyperpolarising current injections. b) Potent hyperpolarisation of four SST+ interneurons during green light illumination $(1.45 \mathrm{~mW})$. c-d) Effects of laser power on network activity. c) Power change between the last half of laser stimulation to baseline against approximate light intensity $(n=17)$. Coloured line represents the average and shaded area the SEM. d) Average peak frequency for half-maximal response of SST+ interneuron photo-inhibition (light intensity for each experiment that changed power-area by half of the maximum response). rmANOVA, $\mathrm{F}(3,48)=19.27$, $\mathrm{p}<0.001 ; * * * \mathrm{p}<0.001$, rmANOVA followed by post-hoc paired t-test for multiple comparisons. Solid brackets represent paired t-tests. 


\section{Rhythmic synchronisation of the hippocampal network by perisomatic and dendritic inhibition}

The experiments using photo-inhibition indicate that the generation of gamma oscillations in hippocampal area CA3 involves the endogenous recruitment of both PV+ and SST+ interneurons. In order to test whether the activation of $\mathrm{PV}+$ or SST+ interneurons is sufficient to synchronise the hippocampal network at gamma frequencies, we next examined cell type-specific photo-stimulation using Channelrhodopsin 2 (ChR2) (Nagel et al., 2003; Boyden et al., 2005). Injection of AAV-ChR2mCherry produced similar expression patterns as Arch in both PV- and SST-Cre mouse lines (Fig. 3a, b). Photo-stimulation of ChR2-expressing PV+ interneurons at $40 \mathrm{~Hz}$ (1 ms pulse width) entrained ongoing oscillations in 14/18 experiments ( $>2 \mathrm{~mW} ; \mathrm{n}=12$ at $5.5 \mathrm{~mW}, \mathrm{n}=6$ at $2.2 \mathrm{~mW}$ - merged due to similar effects; Fig. 3c, f and Supplementary Fig. 3ai). In the remaining 4 out of 18 experiments the ongoing oscillations were not entrained (Fig. 3f; Supplementary Fig. 3aiii). This effect was likely observed due to low ChR2 expression, as pulses with longer width (5 ms) entrained the oscillation in the same experiments (Supplementary Fig. 3b-c). Thus, PV+ interneurons are sufficient to synchronise the hippocampal network at gamma frequencies.

Rhythmic photo-stimulation of SST+ interneurons reliably entrained ongoing oscillations in 19 out of 22 experiments ( $>2 \mathrm{~mW} ; \mathrm{n}=13$ at $5.5 \mathrm{~mW}, \mathrm{n}=9$ at $2.2 \mathrm{~mW}$ - merged due to similar effects; Fig. $3 \mathrm{~d}, \mathrm{~g})$. In the remaining 3 out of 22 oscillations were abolished during $40 \mathrm{~Hz}$ photo-stimulation. These results indicate that transient activation of SST+ dendrite-targeting interneurons is also sufficient to synchronise the hippocampal network at gamma frequencies. Activation of PV+ and SST+ interneurons produced opposite deflections in the pulse-locked waveform of the LFP recorded in the stratum pyramidale (Fig. 3c-e), as might be expected from the somatodendritic profile of their axon terminations. However, activation of SST+ interneurons was sometimes accompanied by an initial fast negative component (Fig. 3e), which was reminiscent of a population spike arising from 
188 the synchronised firing of excitatory cells in the hippocampus (Andersen, Bliss \& Skrede, 1971;

189 Wierenga \& Wadman, 2003), despite the sparsity of SST+ axons in this layer.

190 To study the SST+ induced waveform in isolation, we repeated the same experiment in quiescent 191 slices, perfused only with aCSF. Blue light pulses (1 ms width) at $40 \mathrm{~Hz}$ induced strong pulse-locked 192 field responses with fast-negative deflections, which were resistant to glutamate receptor blockers 193 (Supplementary Fig. 3d-f), but were followed by a glutamate receptor-mediated positive deflection.

194 The fast-negative deflections did not appear to reflect fast GABAergic transmission, as application 195 of $G_{A B A_{A}}$ receptor $\left(G A B A_{A} R\right)$ blockers lead to light-induced epileptiform bursts $(n=4$; Supplementary Fig. $3 g$ ). These results suggest that SST+ interneuron photo-activation generates network excitation, that is not mediated through $\mathrm{GABA}_{A} \mathrm{Rs}$, at the onset of light illumination. We did not observe ChR2 expression in CA3 pyramidal neurons during intracellular recordings $(n=18$, supplementary Fig. 5e), although there have been reports of off-target expression in SST+ interneurons of juvenile animals (Taniguchi et al., 2011). An alternative possibility is that robust 201 activation of a dense plexus of SST+ axons in the dendritic layers is sufficient to induce spiking in pyramidal neurons via ephaptic coupling (Ferenczi et al., 2016a). Either scenario makes it difficult to 203 interpret the results of pulsed stimulation in the SST-ChR2 mice, but any electrically-mediated bystander effects are likely to occur during stimulus onset (maximal hypersynchrony), and may be less relevant during more sustained patterns of stimulation. 
a.

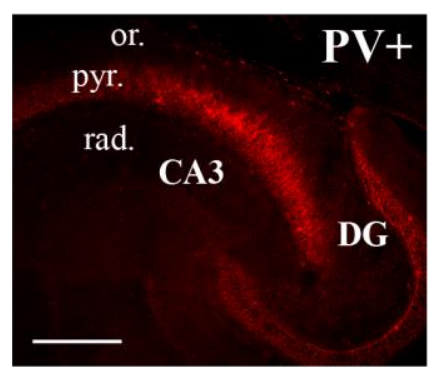

b.

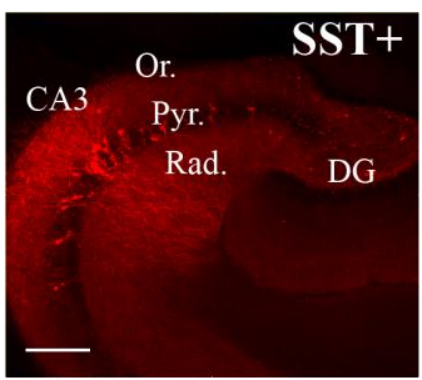

C. $160 \mu \mathrm{V}$
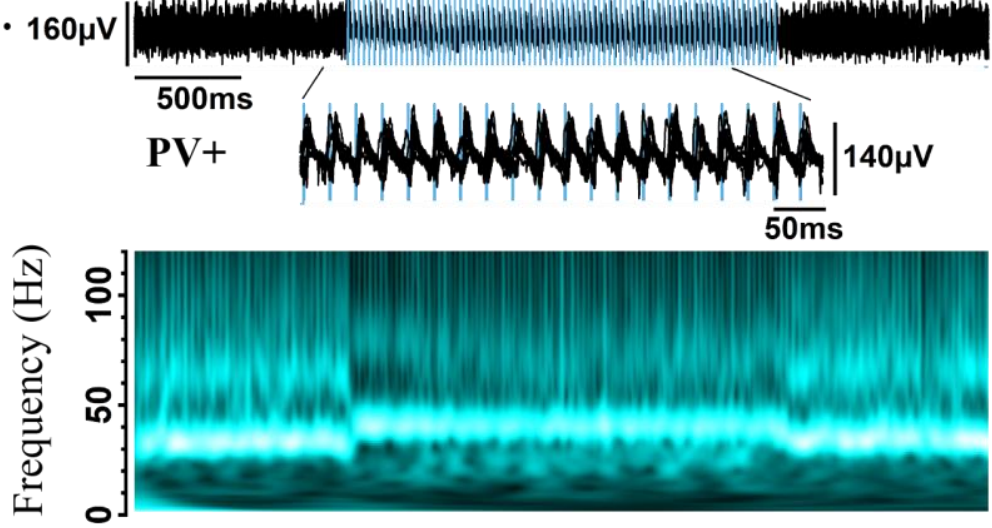

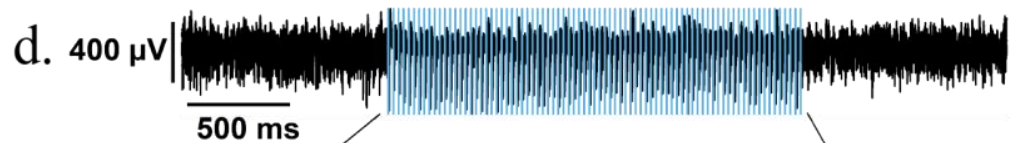

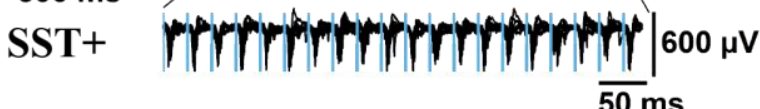

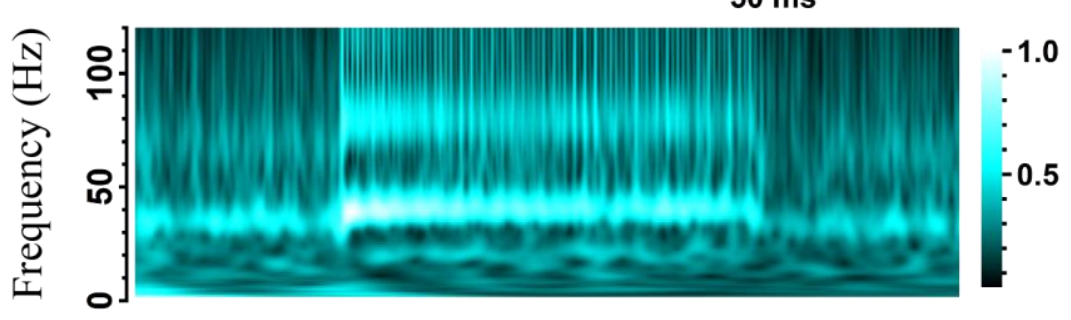

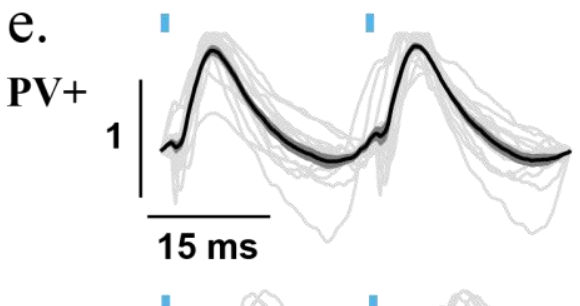

SST+

f.

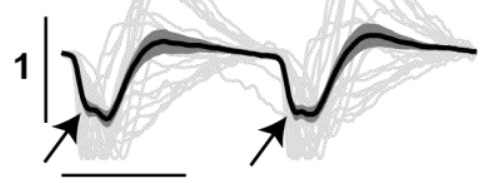

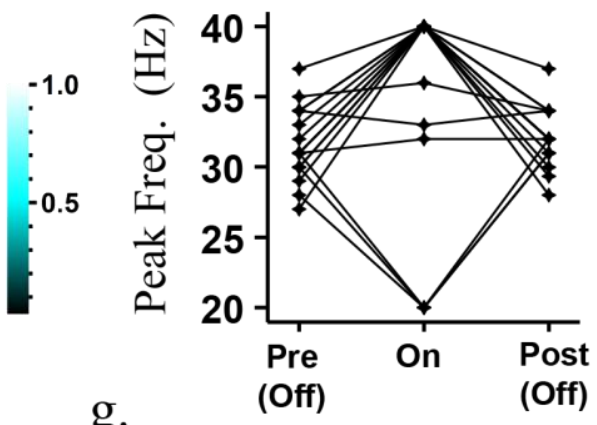

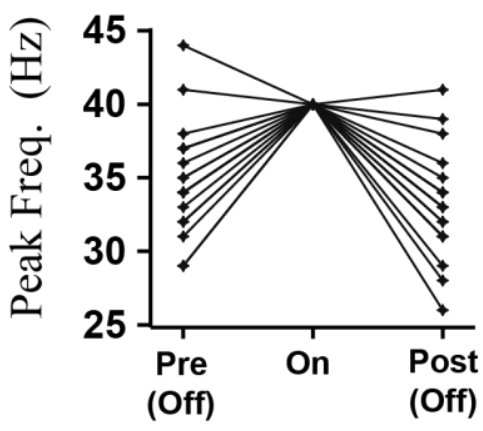

Figure 3: Rhythmic photo-stimulation of either PV+ or SST+ interneurons entrains Cch-induced gamma oscillations. Confocal image of ventral hippocampus (350 $\mu \mathrm{m}$ slice) from a: PV-cre mice and b: SST-cre mice with mCherry-ChR2 expression. CA3 = Cornu Ammonis 3, DG = Dentate Gyrus, Pyr. $=$ stratum Pyramidale, Rad. $=$ stratum Radiatum, Or. $=$ Stratum Oriens. Scale bar $=200 \mu \mathrm{m} . \mathrm{c}$ and d) Top: Representative LFP recording from CA3 area illustrating the entrainment of Cch-induced oscillations to $40 \mathrm{~Hz}$ light pulses in c: PV-cre and d: SST-cre mice expressing mCherry-ChR2 (1ms pulse width; blue light illumination at $5.5 \mathrm{~mW}$ ). Middle: Zoomed recordings during pulse stimulation. Bottom: Magnitude component of the wavelet transform normalised by its maximum value. Brighter colours represent larger gamma power. e) Normalised average waveform following two consecutive pulses at $40 \mathrm{~Hz}$ and $1 \mathrm{~ms}$ pulse width from each experiment (PV+: $\mathrm{n}=15 / 18$; SST+: $\mathrm{n}=19 / 22$ ). Black line is the population average, grey lines represent individual experiments and dark-grey shaded area is the SEM Black. Arrows indicate initial negative peak. $\mathrm{f}, \mathrm{g}$ ) Peak frequency of oscillation before (Pre (Off)), during (On) and after (Post (Off)) light stimulation in $\mathrm{f}: \mathrm{PV}+(\mathrm{n}=18 / 18)$ and $\mathrm{g}: \mathrm{SST}+(\mathrm{n}=19 / 22)$ experiments (note experiments entrained at $20 \mathrm{~Hz}$ reflect suppression of alternate gamma cycle - see suppl. Fig. 3aii). Black lines represent single experiments. 


\section{Supplementary Figure 3 (Supporting Figure 3)}
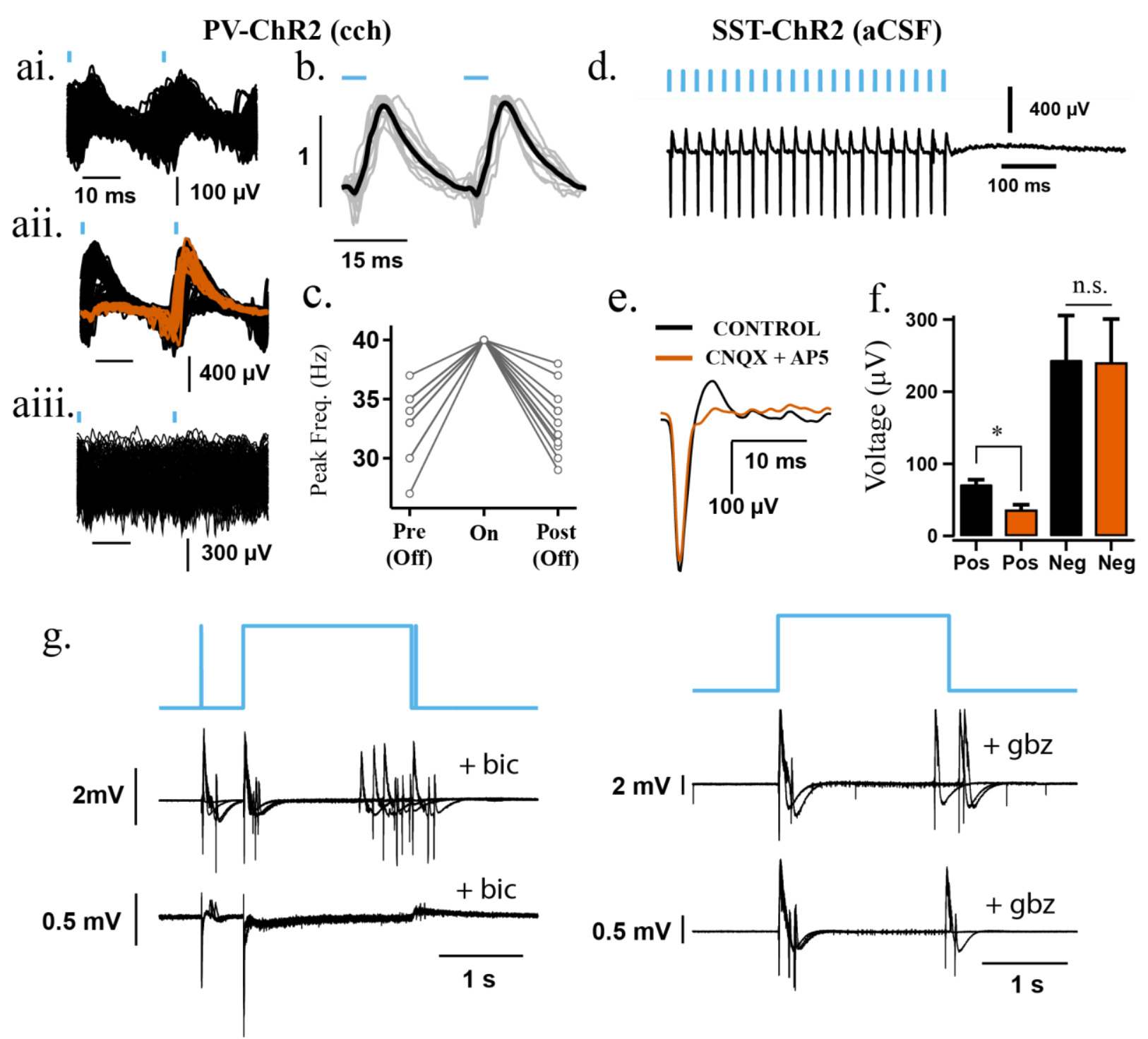

Supplementary Figure 3: a-c) Responses to pulse stimulation in slices with Cch-induced gamma oscillations from PV-ChR2 mice. a) Extracted waveforms following two consecutive pulses at $40 \mathrm{~Hz}$ (1 $\mathrm{ms}$ ) from one experiment showing i) strong entrainment, ii) entrainment of half of the cycles and inhibition of the other half, and iii) no entrainment. Coloured lines represent a subset of extracted waveforms. b) Average waveform following two consecutive pulses at $40 \mathrm{~Hz}$ with pulse widths of $5 \mathrm{~ms}(\mathrm{n}=13 / 13)$. Black line is the population average, grey lines represent individual experiments and dark-grey/error bars shaded area is the SEM. c) Peak frequency of oscillation before (Pre (Off)), during (On) and after (Post (Off)) light stimulation at $40 \mathrm{~Hz}$ with pulse widths $5 \mathrm{~ms}(\mathrm{n}=13 / 13)$. Note that the slices with $20 \mathrm{~Hz}$ induction were not tested with $5 \mathrm{~ms}$ pulse width. $\mathrm{d}-\mathrm{g}$ ) Responses to pulse stimulation in slices from SSTChR2 mice without cholinergic activation (aCSF). d) Representative LFP recording from CA3 area illustrating field responses during $40 \mathrm{~Hz}$ light pulses in the absence of Cch (1ms pulse width- $5.5 \mathrm{~mW})$. e) Average waveform obtained with $1 \mathrm{~ms}$ pulse width at $40 \mathrm{~Hz}$ from one experiment before (black line) and after application of $20 \mu \mathrm{M}$ CNQX and $40 \mu \mathrm{M}$ AP5. In one experiment only $20 \mu \mathrm{M}$ CNQX was applied but exhibited the same effect. f) The magnitude of negative and positive peaks before and after iGluR blocker application $(\mathrm{n}=4)$. iGluR blockers used: $20 \mu \mathrm{M}$ CNQX, $40 \mu \mathrm{M}$ AP5, $\mathrm{n}=3 ; 20 \mu \mathrm{M}$ CNQX, $\mathrm{n}=$ 1. Positive peak before (MaxC) and after drug (MaxD) application $(t=3.49, p=0.03$, paired t-test). Negative peak before $(\mathrm{MinC})$ and after drug $(\mathrm{MinD})$ application $\left(\mathrm{t}=-0.61, \mathrm{p}=0.58\right.$, paired $\mathrm{t}$-test). ${ }^{*} \mathrm{p}$ $\langle 0.05$, n.s. $\mathrm{p}\rangle=0.05$. g) Induction of epileptiform bursts during photo-stimulation of SST+ interneurons following $\mathrm{GABA}_{\mathrm{A}} \mathrm{R}$ blockage ( $\mathrm{n}=2$ at $20 \mu \mathrm{M}$ bicuculine (bic), $\mathrm{n}=2$ at $10 \mu \mathrm{M}$ gabzine (gbz)). 


\section{Sustained activation of PV+ interneurons suppresses Cch-induced gamma}

209

\section{oscillations}

We used two patterns of sustained activation, light steps and fully-modulation sine waves at $8 \mathrm{~Hz}$, and tested these in slices from PV-ChR2 mice. In a subset of light step experiments, we recorded ongoing gamma oscillations in the LFP whilst tonically driving PV+ interneurons at increasing strengths across trials (by changing the levels of blue light illumination, $10-5500 \mu \mathrm{W}$ ). The change in power between baseline and light activation period was measured at each light intensity level. We then obtained the response level at which the power changed by half of the maximum for each experiment (half-maximal response). For half-maximal response trials, photo-activation of PV+ interneurons ( 2 seconds) consistently decreased the power-area $(0.52+/-0.016$ compared to baseline, $t=-29.56, p<0.01$, one-sample t-test; Fig. 4a-c, e) and increased the peak frequency (from $32.70+/-0.793 \mathrm{~Hz}$ (baseline) to $38.76+/-1.094 \mathrm{~Hz}, \mathrm{t}=8.21, \mathrm{p}<0.01$, paired t-test; Fig. 4d, f). Furthermore, there was a progressive decrease in power $(r=-0.84, n=121$ values, $t=17.00, p<$ $0.01)$ and increase in frequency $(r=0.49, n=100 / 121$ values, $t=5.60, p<0.01)$ as the light intensity increased (Fig. 4g-h). In order to estimate the maximal effect of PV+ interneuron stimulation, we pooled experiments using strong light intensity illumination $(>2 \mathrm{~mW}$, including cases where light intensity-response curves were not assessed; $\mathrm{n}=14$ at $5.5 \mathrm{~mW}, \mathrm{n}=9$ at $2.2 \mathrm{~mW}$ ). Overall, strong light illumination caused a substantial decrease in the normalised power-area $(0.09+/-0.029, \mathrm{t}=$ $31.07 \mathrm{p}<0.01$, one-sample t-test; Fig. $4 \mathrm{i}, \mathrm{j}$ ) and abolished the oscillations in most experiments (17/23). These results indicate that progressive up-regulation of PV+ interneuron activity decreases gamma power and increases the frequency until the rest of the hippocampal network is fully silenced.

Interneurons have been shown to be particularly susceptible to depolarisation block (Herman et al., 2014). In order to ensure that these effects were not caused from impaired action potential 
generation in PV+ interneurons (i.e. depolarisation block), as we recorded spiking activity using a linear multi-electrode array (MEA) during Cch-induced oscillations. PV+ interneurons (spike width: 0.49 +/- $0.04 \mathrm{~ms}$ ) maintained spiking activity during sustained illumination (5.5 mW; Median sustained activation index $[\mathrm{IQR}]=0.87[0.46,1], \mathrm{Z}=171, \mathrm{p}<0.001, \mathrm{n}=18$, one-sample Wilcoxon signed rank test; analysis performed on last second of trial), and this was associated with decreased activity of regular spiking (RS; $-0.72[-0.92,-0.40] ; Z=2, p<0.001, n=53$, one-sample Wilcoxon signed rank test; $z=65.7, p<0.001$ cf. PV+ interneurons, Kruskal-Wallis Test followed by posthoc Dunn's test with Bonferroni correction for multiple comparisons) and fast-spiking cells (FS; $-0.52[-0.82,-0.12] ; Z=141$ $p<0.001, n=49$, one-sample Wilcoxon signed rank test; $z=50.1, p<0.001 \mathrm{cf}$. PV+ interneurons, Kruskal-Wallis Test followed by posthoc Dunn's test with Bonferroni correction for multiple comparisons) (Supplementary Fig.4). These results are consistent with increased PV+ interneuron activity during light illumination that leads to reduced activity in hippocampal principal cells.

During $8 \mathrm{~Hz}$ sinusoidal modulation of PV+ interneurons, the instantaneous gamma magnitude, assessed using the Hilbert transform, was found to be negatively correlated with light intensity in agreement to light step experiments (across all experiments, Pearson correlation, mean $r=-0.51+/$ 0.04, $\mathrm{t}>23.2, \mathrm{p}<0.01, \mathrm{n}=12$ ) (Suppl. Fig.4d). During MEA recordings, spike rates of PV+ interneurons correlated positively with theta-frequency changes in light intensity (Median rank correlation coefficient $[\mathrm{IQR}]=0.75[0.55,0.83], \mathrm{Z}=120, \mathrm{p}=0.001, \mathrm{n}=15$, one-sample Wilcoxon signed rank test), while negative correlations were found for the spike rates of RS $(-0.19[-0.37,-0.09]$; $Z=100, p<0.001, n=43$, one-sample Wilcoxon signed rank test; $z=48.3, p<0.001$ cf. PV+ interneurons, Kruskal-Wallis Test followed by posthoc Dunn's test with Bonferroni correction for multiple comparisons) and FS cells $(-0.19[-0.39,-0.01] ; Z=232, p=0.006, n=42$, one-sample Wilcoxon signed rank test; $z=45.7, p<0.001$ cf. PV+ interneurons, Kruskal-Wallis Test followed by posthoc Dunn's test with Bonferroni correction for multiple comparisons). These findings indicate that a transient 
bioRxiv preprint doi: https://doi.org/10.1101/595546; this version posted April 1, 2019. The copyright holder for this preprint (which was not certified by peer review) is the author/funder, who has granted bioRxiv a license to display the preprint in perpetuity. It is made available under aCC-BY 4.0 International license.

256 increase in PV+ interneuron activity causes a rapid and reversible decrease in the power of the Cch-

257 gamma oscillations and firing rates of other neurons. 
a.

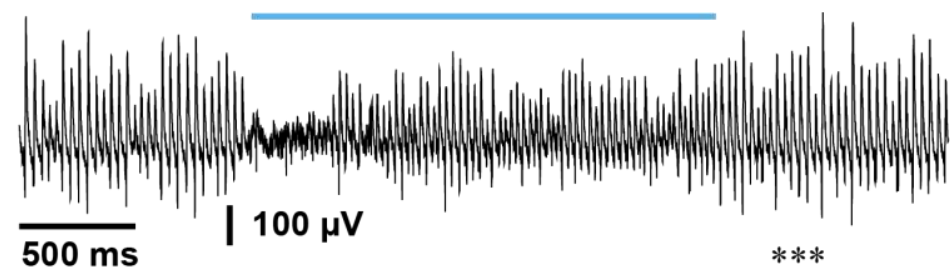

c.

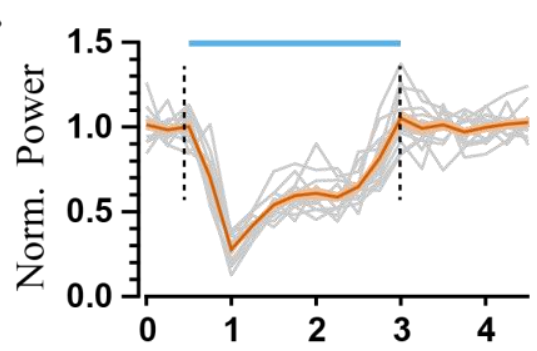

d.

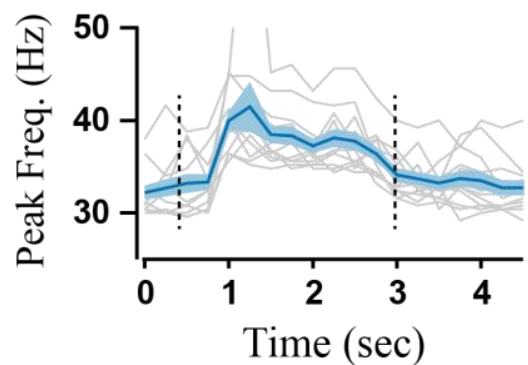

i.

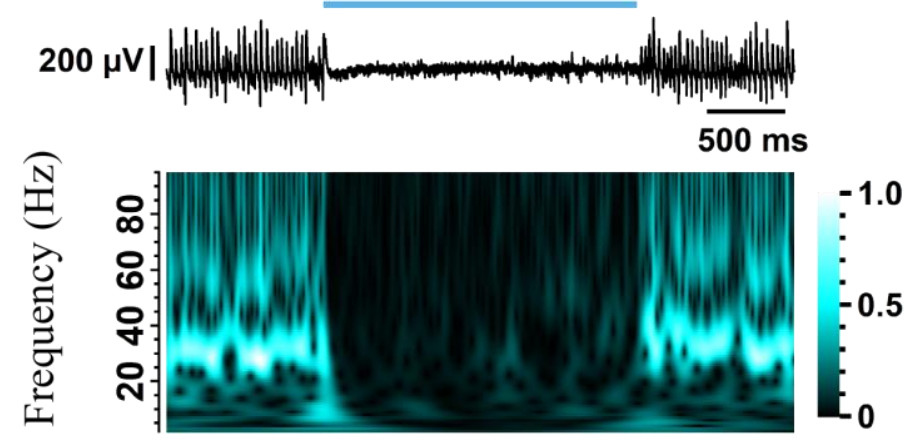

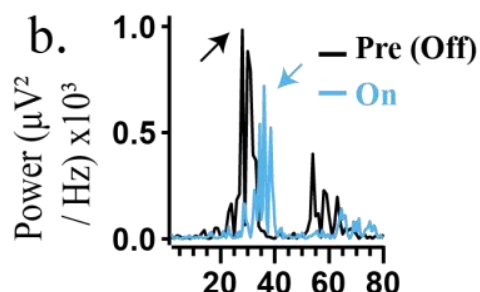

Frequency $(\mathrm{Hz})$

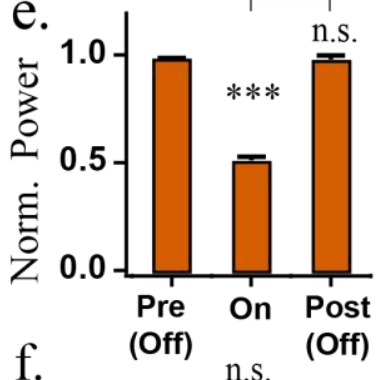

f.
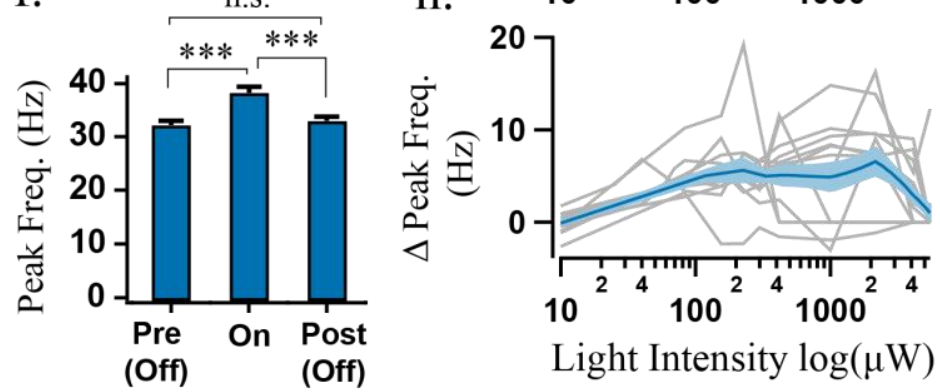

Light Intensity $\log (\mu \mathrm{W})$

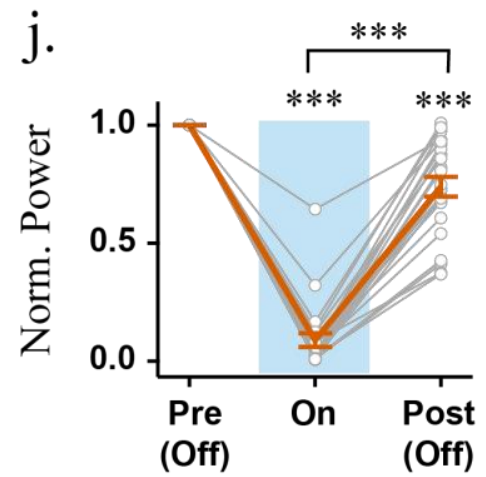

Figure 4: Sustained photo-excitation of PV+ interneurons decreases the power and increases the frequency of Cch-induced gamma oscillations. a) Representative LFP recordings from CA3 area illustrating effect of $\mathrm{PV}+$ interneurons photo-excitation $(155 \mu \mathrm{W})$ on gamma oscillations along with $\mathrm{b})$ its respective power spectrum (arrows indicate power spectrum peaks). c) Power-area normalised to baseline and d) peak frequency of the oscillation calculated in 0.5 second bins across experiments $(n=12)$. e) Average change in power-area during (On) and after light stimulation (Post (Off)) normalised to baseline (Pre (Off)). f) Average peak frequency; rmANOVA: $\mathrm{F}(1.14,12.50)=44.14, \mathrm{p}<0.001$. g) Power-area change and $\mathrm{h}$ ) frequency difference between light stimulation period and baseline plotted against light intensity $(n=12)$. i) Top: Representative LFP recording from CA3 area illustrating the collapse of Cch-induced oscillations in response to strong and sustained blue light illumination $(5.5 \mathrm{~mW})$. Bottom: Magnitude component of the wavelet transform normalised by its maximum value. $\mathrm{j}$ ) Average change in power-area upon strong and sustained blue light illumination ( $\mathrm{n}$ total $=23>2 \mathrm{~mW}: \mathrm{n}=14$ at $5.5 \mathrm{~mW}$ and $\mathrm{n}=9$ at $2.2 \mathrm{~mW}$ ). Changes in peak frequency were analysed using rmANOVA, followed by post-hoc paired t-tests with correction for multiple comparisons. $* \mathrm{p}<0.05, * * \mathrm{p}<0.01, * * * \mathrm{p}<0.001$, n.s. $\mathrm{p}\rangle=0.05$. Solid brackets represent paired t-tests and standalone star symbols represent one-sample t-test versus normalised baseline. Grey lines represent single experiments, error bars and shaded area are SEM and coloured line the population average. 


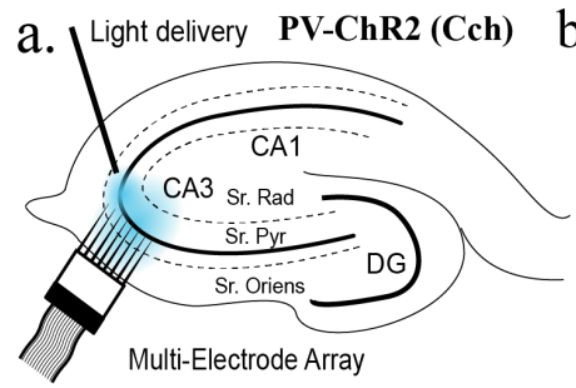

c.

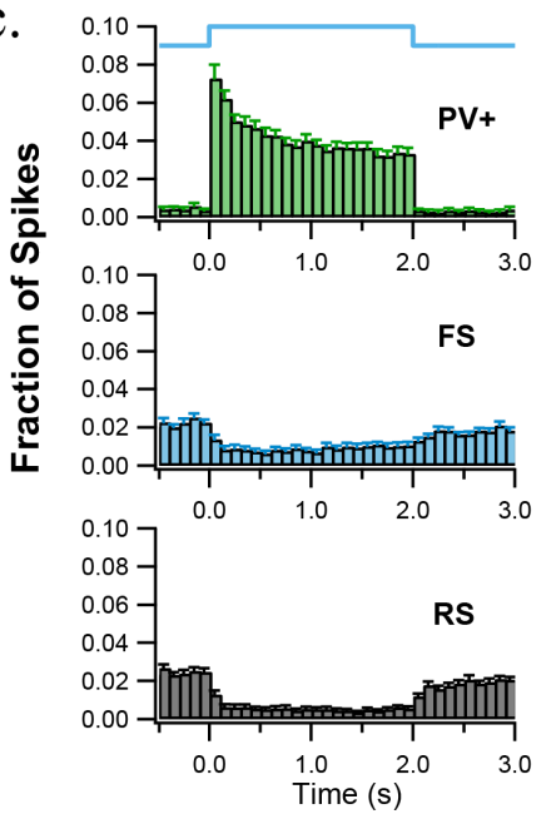

b.

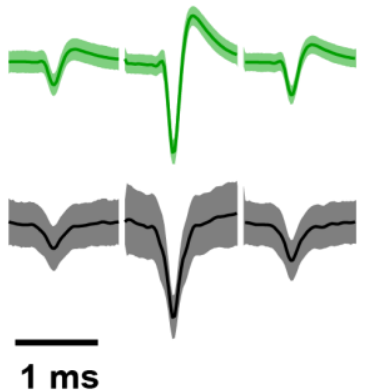

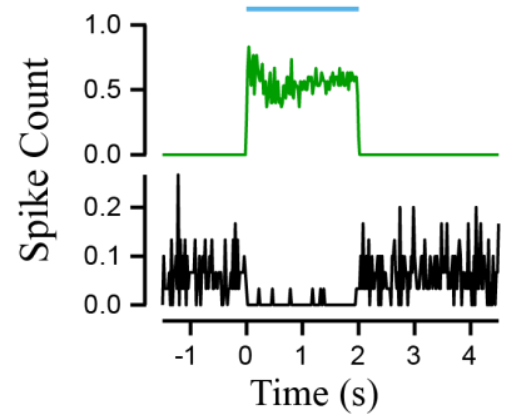

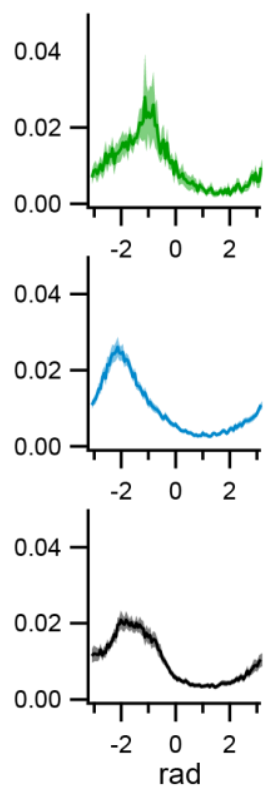

d.

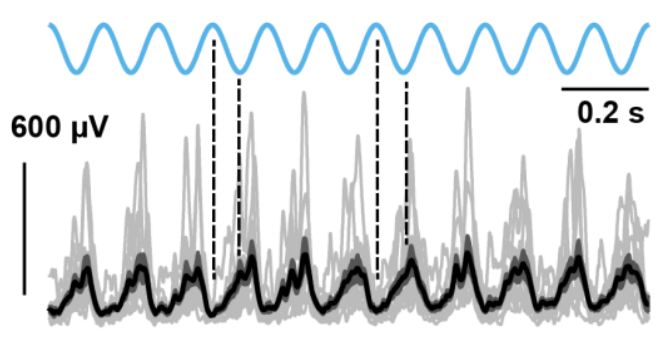

e.

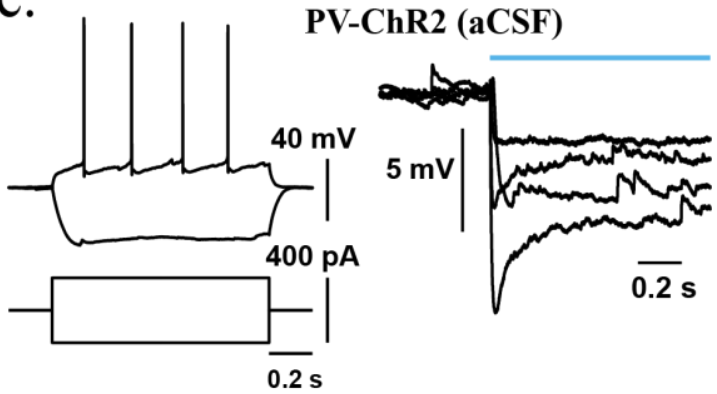

Supplementary Figure 4 (a-c): Multi-unit recordings during PV+ interneuron sustained photo-excitation in hippocampal slices with Cch-induced gamma oscillations. a) Schematic diagram of the hippocampus illustrating MEA recordings during blue light illumination $(5.5 \mathrm{~mW})$ in CA3. b) Left - Representative average spike waveforms, Right - spike histograms during sustained light illumination. (green FS single units, black RS multi-unit). c) Mean spike time histograms (left) and spike phase histograms (right) of photo-tagged PV+, FS and RS cells. Shaded regions represent standard deviation. The PV+ interneurons fired at a significantly later phase of the oscillation than the RS cells $(F(2,55)=5.36, p=0.007$, Two-sample Hotelling test). d) Instantaneous amplitude of the Hilbert transform during theta photo-activation (1 mW) overlaid across experiments (grey traces, $n=12$ ), black represents the mean and dark grey the SEM. Dotted lines illustrate that high light intensity decreased gamma power. e) Intracellular recordings from pyramidal cells in aCSF, showing responses to current steps (left) and hyperpolarisation in response to PV+ interneuron photo-activation $(n=4)$. 


\section{Sustained activation of SST+ interneurons induces fast gamma}

\section{oscillations}

We obtained the light intensity response curves with light steps in slices from SST-ChR2 mice and observed similar results as in PV-ChR2 experiments. Sustained light illumination decreased the power $(0.49+/-0.029, t=17.53, p<0.01$, one sample t-test; Fig. 5a-c, e) and increased the frequency at half-maximal response (from $34.08+/-0.954 \mathrm{~Hz}$ during baseline period to $38.17+/-1.400 \mathrm{~Hz}, \mathrm{t}=$ 3.658, $p=0.011$, paired t-test; Fig. $5 d, f)$. Moreover, as the light intensity increased, the power progressively decreased $(r=-0.66, n=107$ values, $t=9.11, p<0.01 ;$ Fig. $5 g)$, and frequency progressively increased $(r=0.71, n=56$ out of 107 values, $t=7.41, p<0.01$; Fig. $5 \mathrm{~h})$. It is perhaps not surprising that excitatory networks can be suppressed by photo-activation of GABAergic interneurons. However, different responses were revealed when we assessed the effects of strong photo-activation of SST+ interneurons on Cch-induced gamma oscillations (light-intensity response curves where performed in a subset of experiments; $n=18$ slices at $5.5 \mathrm{~mW}$ and $\mathrm{n}=13$ slices at 2.2 $\mathrm{mW}$, merged). Consistent with PV-ChR2 step experiments, the gamma power was reduced during light stimulation when compared to baseline period $(0.34+/-0.150, t=-4.39, p<0.01$, one sample t-test; Fig. 4.6b) and in approximately half of the experiments, gamma oscillations were fully abolished ( $n=16 / 31$ slices). In contrast, in experiments where the oscillations persisted, their frequency increased strongly from $34.63+/-0.836 \mathrm{~Hz}$ during baseline to 62.75 +/- $4.921 \mathrm{~Hz}$ during light illumination ( $n=15 / 31$ slices; $t=5.61, p<0.01$, paired $t$-test; Fig $5 i-k$ ). These fast gamma oscillations occurred most reliably in slices that the light-intensity response curves were not obtained. In order to test if SST+ interneuron photo-activation alone is sufficient to induce oscillations, as opposed to simply increasing the frequency of ongoing activity, we repeated the same experiments in the absence of Cch. Sustained photo-activation of SST+ interneurons induced de novo oscillations in the fast gamma-band range with peak frequency of $80.5+/-2.48 \mathrm{~Hz}(12 / 16$ 
284 slices; Fig. 6a, c). Isolating the CA3 area from DG did not prevent the generation of de novo 285 oscillations ( $\mathrm{n}=3$ slices).

286 Furthermore, sinusoidal light activation at $8 \mathrm{~Hz}$ (theta photo-activation) also induced robust 287 oscillations with higher peak frequency than the tonic activation $111.2+/-3.15 \mathrm{~Hz}(13 / 17$ slices; $\mathrm{t}=$ 288 7.64, $p<0.001$, two-sample t-test; Fig. 6b, d-e). This is consistent with previous experiments 289 showing that transient light activation induces higher frequency oscillations than sustained 290 illumination (Butler et al., 2016; Betterton et al., 2017). Furthermore, the power $(r=0.67, n=70$ 291 values, $t=7.52 p<0.01)$ and frequency $(r=0.77, n=48 / 70$ values, $t=8.20, p<0.01)$ of the de novo 292 oscillations progressively increased as the light intensity of theta photo-activation was elevated (Fig. 293 6f). This monotonic increase in peak frequency contrasts with the properties of oscillations induced 294 by photo-activation of principal cells in the hippocampus, where the frequency of the oscillations 295 remains relatively constant within the slow gamma band across light intensities (Butler et al., 2016; 296 Betterton et al., 2017; Butler, Hay \& Paulsen, 2018). Therefore, SST+ interneuron photo-activation 297 in CA3 appears to induce a distinct type of gamma activity. 
a.

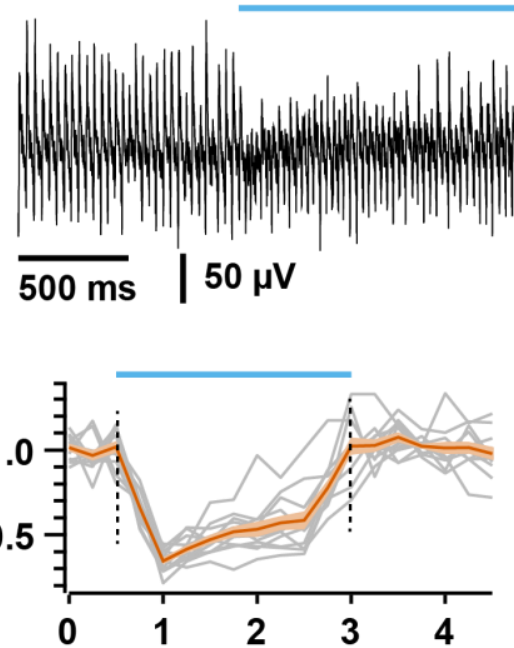

d.

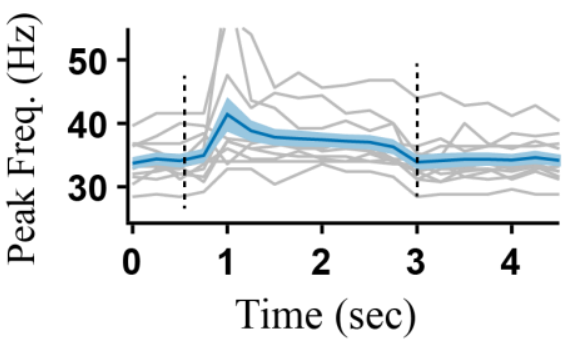

i.
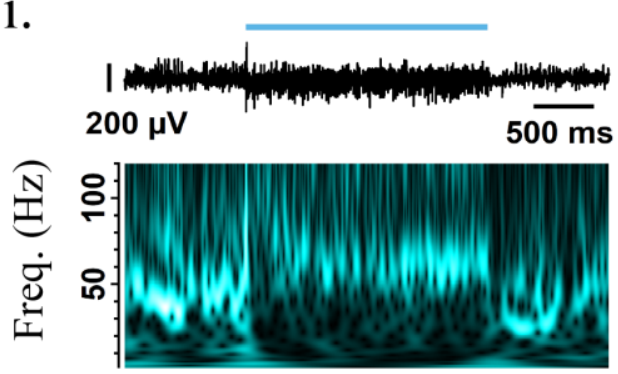

e.
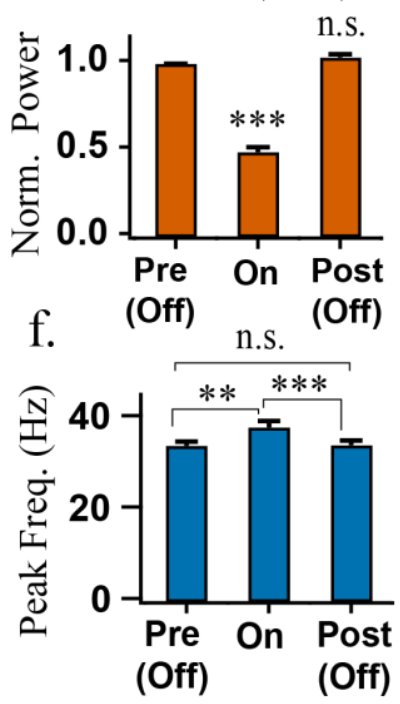

$\mathrm{j}$.

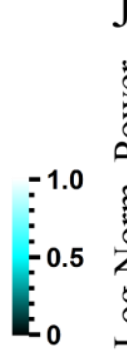

b.
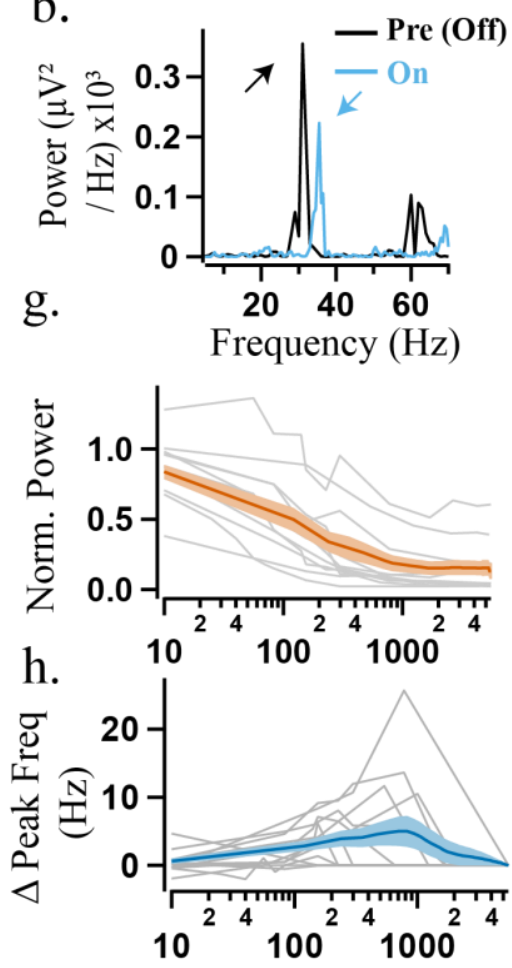

Light Intensity $\log (\mu \mathrm{W})$

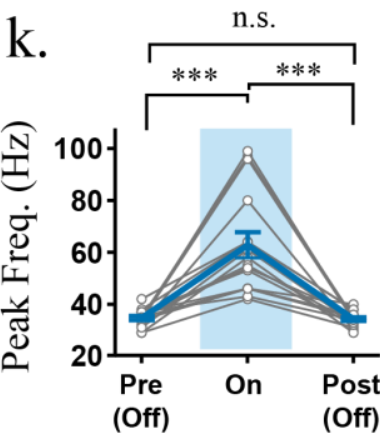

Figure 5: Sustained photo-excitation of SST+ interneurons decreases the power and increases the frequency of Cch-induced gamma oscillations but can also induce high-frequency oscillations. a) Representative LFP recordings from CA3 area illustrating effect of SST+ interneurons photo-excitation $(155 \mu \mathrm{W})$ on gamma oscillations along with $\mathrm{b}$ ) its respective power spectrum (arrows indicate power spectrum peaks). c) Power-area normalised to baseline and d) peak frequency of the oscillation calculated in 0.5 second bins across experiments $(\mathrm{n}=12)$. e) Average change in power-area during (On) and after light stimulation (Post (Off)) normalised to baseline (Pre (Off)). f) Average peak frequency; rmANOVA: F(1.05, 11.59) $=15.05, \mathrm{p}=0.002$. g) Power-area change and h) frequency difference between light stimulation period and baseline plotted against light intensity $(n=12)$. i) Top: Representative LFP recording from CA3 area illustrating the induction of high-frequency oscillations in response to strong and sustained blue light illumination $(5.5 \mathrm{~mW})$. Bottom: Magnitude component of the wavelet transform normalised by its maximum value. j) Normalised power of Cch-oscillations during $\mathrm{SST}+$ interneurons cell photo-activation $(\mathrm{n}=31) \mathrm{k})$ Peak frequency of oscillations that were not abolished from strong light illumination ( $\mathrm{n}$ remaining $=16 / 31: \mathrm{n}=4$ at $5.5 \mathrm{~mW}$ and $\mathrm{n}=12$ at $2.2 \mathrm{~mW}$ ); rmANOVA: $\mathrm{F}(1.03$, $15.39)=31.45, \mathrm{p}<0.001$. Changes in peak frequency were analysed using rmANOVA, followed by post-hoc paired t-tests with correction for multiple comparisons. $* \mathrm{p}\left\langle 0.05,{ }^{*} \mathrm{p}<0.01\right.$, ${ }^{* *} \mathrm{p}<\langle 0.001$, n.s. $\mathrm{p}\rangle=0.05$. Solid brackets represent paired t-tests and standalone star symbols represent one-sample t-test versus normalised baseline. Grey lines represent single experiments, error bars and shaded area are SEM and coloured line the population average. 
The fast gamma oscillations that emerge during sustained photo-activation of SST+ interneurons could reflect the intrinsic synchronisation of SST+ networks, but there are a number of possible scenarios in which this stimulation paradigm could lead to the activation of other hippocampal microcircuits involving network excitation. Depolarising GABA could contribute to recruitment of postsynaptic targets, but perforated patch recordings from hippocampal cells in stratum pyramidale (aCSF only) showed that they were hyperpolarized by light illumination (Supplementary Fig. 5f-g). Alternatively, network excitation and oscillogenesis could emerge following depolarisation block of SST+ interneurons, and subsequent disinhibition, but direct photo-inhibition of SST+ interneurons was not able to generate de novo oscillations (Supplementary Fig. 5a-b). However, the power of the light-induced oscillations was markedly reduced following block of either fast excitation or inhibition, and whole cell recordings in putative principal cells indicated that they received weak excitatory postsynaptic currents (EPSCs) throughout light illumination (Supplementary Fig. 5c-e, hj). This suggests that the light-induced oscillations recorded in LFP do not emerge solely from the activity of SST+ interneurons.

To directly test if photo-excitation of SST+ interneurons leads to a dominant effect of depolarisation block during ongoing gamma oscillations, and whether photo-excitation is associated with net increases or decreases in the spiking activity of other neurons in the network, we performed MEA recordings. We found that SST+ interneurons (spike width: $0.69+/-0.03 \mathrm{~ms}$ ) displayed increased activity throughout the course of step stimulation (Median sustained activation index $[\mathrm{IQR}]=0.90$ [0.76, 0.98], $Z=2346, p<0.001, n=68$, one-sample Wilcoxon signed rank test; Fig. 6j-top), and faithfully followed the $8 \mathrm{~Hz}$ sine stimulation (Median rank correlation coefficient $[\mathrm{IQR}]=0.63[0.52$, 0.72], $Z=1484, P<0.001, n=54$, one-sample Wilcoxon signed rank test; Fig. $6 \mathrm{j}$-middle). All but 3 of the SST+ interneurons recorded were significantly phase-coupled to the induced fast gamma-frequency oscillations ( $p<0.05$, Rayleigh test), with a mean spike phase of $-2.0[-2.1,-1.8]$ radians (second-order mean [95\% confidence intervals]; $n=65$; Fig. 6j-bottom). The RS and FS cells showed significantly 
weaker modulation (Fig. 6j), but did not appear to be suppressed as in the PV-ChR2 experiments, and rather showed an insignificant trends towards both increased activity during step illumination (Median sustained activation index [IQR]; RS: $0.12[-0.08,0.54], Z=50, p=0.13, n=11 ; F S: 0.49$ [-0.14, theta-frequency changes in light intensity (Median rank correlation coefficient [IQR]; RS: 0.26 [-0.07, $0.53], Z=49, p=0.16, n=11 ; F S: 0.28[0.09,0.57](Z=25, p=0.06, n=7$, one-sample Wilcoxon signed rank tests). The majority of RS (8/11) and FS cells (4/7) were also significantly phase-coupled to the lightinduced fast gamma oscillations ( $p<0.05$, Rayleigh test), but did not show a consistent mean firing phase (RS: $F(2,8)=2.3, p=0.18 ; F S: F(2,2)=4.5, p=0.19$; parametric second-order analysis (Zar, 1999)). oscillations is a robust increase in the spiking of SST+ interneurons.

To explore whether the recruitment of SST+ interneurons might differ between step and theta stimulation, we analysed the maximum spike rates in the second half of the stimulation trials (20 ms bins). The maximum spike rates during theta stimulation were significantly higher than during the step stimulation $(Z=148, p<0.001, n=54$; Wilcoxon signed rank test). As theta stimulation induced faster gamma oscillations than step stimulation (see Fig. 6e), this further suggests that the frequency of fast gamma oscillations depends on the overall levels of SST+ interneuron excitation. 

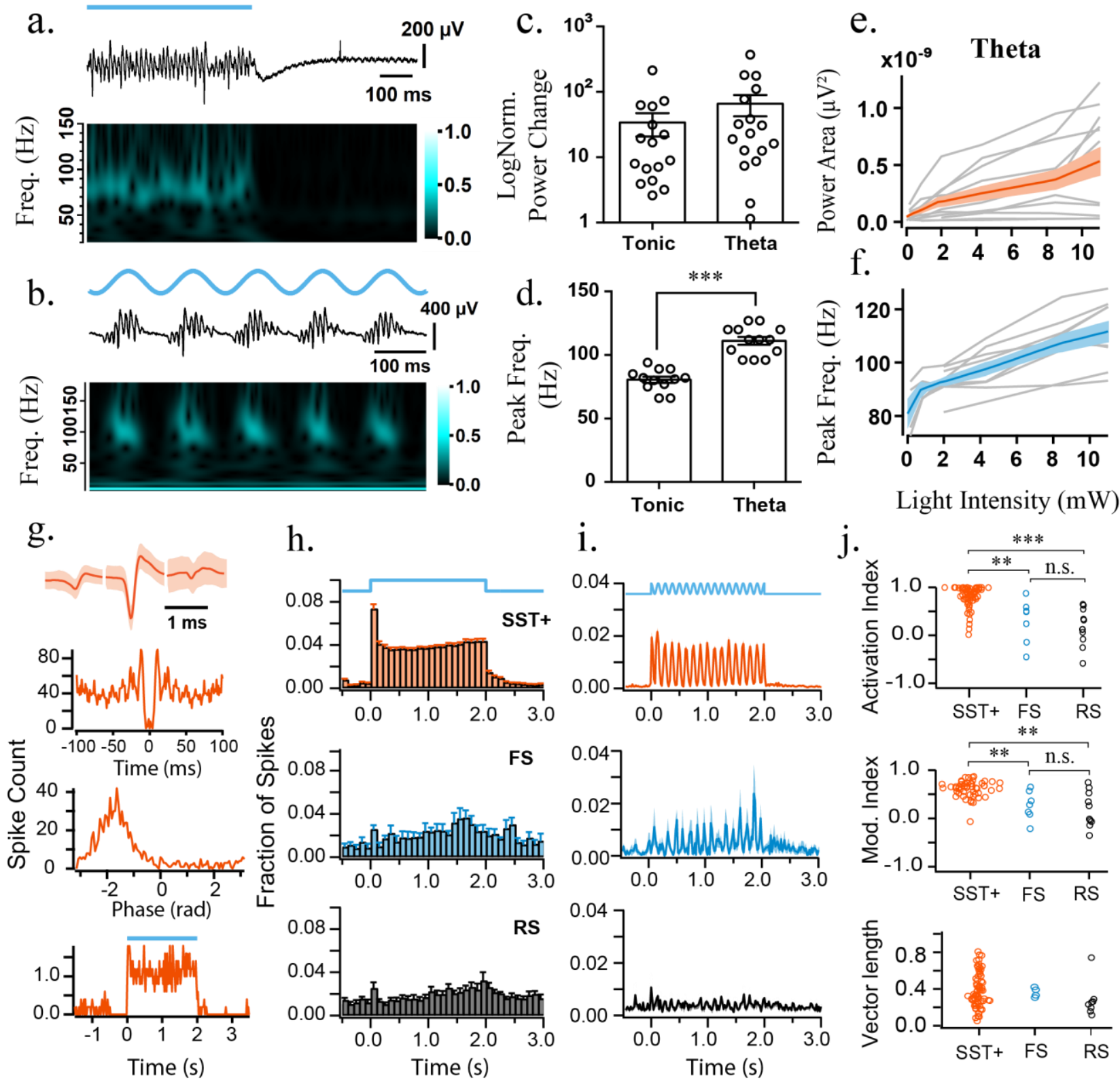

Light Intensity $(\mathrm{mW})$
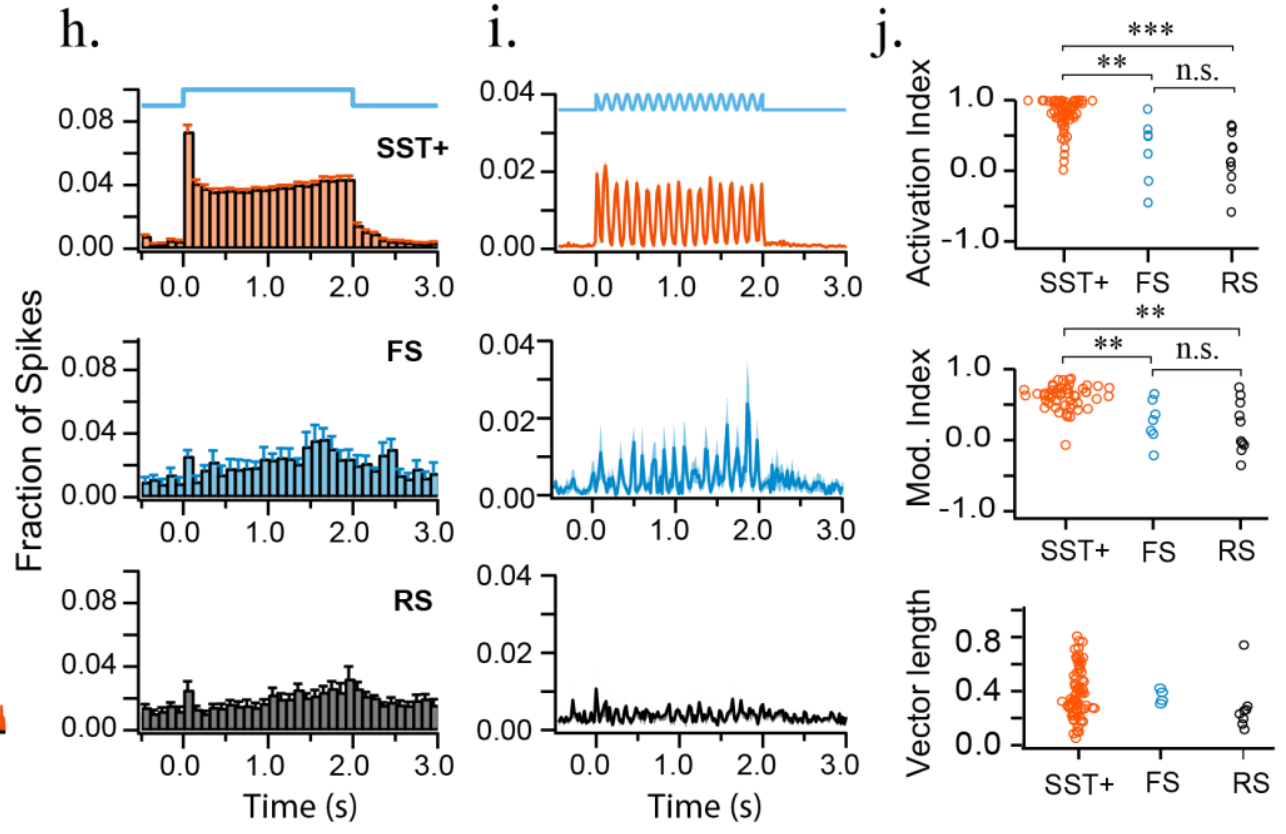

Figure 6: Photo-activation of SST+ interneurons induces de novo oscillations in the absence of Cch. a, b) Top: Representative LFP recording from CA3 area illustrating induction of high-frequency oscillations by a) constant and b) theta blue light illumination $(10 \mathrm{~mW})$. Bottom: Magnitude component of the wavelet transform normalised by its maximum value; brighter colours represent larger magnitude. c) Log power change compared to baseline during constant $(n=16)$ and theta blue light illumination $(n=17)$. d) Peak frequency of the de novo oscillations is higher when induced by theta when compared to tonic stimulation; two-sample t-test, $* * * p<0.001$. Grey lines/markers represent single experiments, black line is the population average and error bars are the SEM. e) Power-area and f) frequency during light stimulation period plotted against light intensity of theta photo-activation $(\mathrm{n}=12)$. The black line is the average response and the darkgrey shaded area represents SEM. (g-i) Multi-unit recordings during SST+ interneuron sustained photoexcitation g) Putative opto-tagged SST+ average spike waveform, autocorrelation, phase and step histogram during sustained light illumination. h) step histogram, i) sinusoidal histogram of photo-tagged SST+, FS and RS cells. Shaded regions represent standard deviation. j) top to bottom: sustained activation index, modulation index and vector length. Kruskal-Wallis Test was followed by posthoc Dunn's test with Bonferroni correction for multiple comparisons. ${ }^{*} \mathrm{p}<0.05$, ${ }^{*} \mathrm{p}<0.01$, $* * \mathrm{p}<<0.001$, n.s. $\mathrm{p}>=0.05$. 


\section{Supplementary figure 5 (Supporting figure 6)}

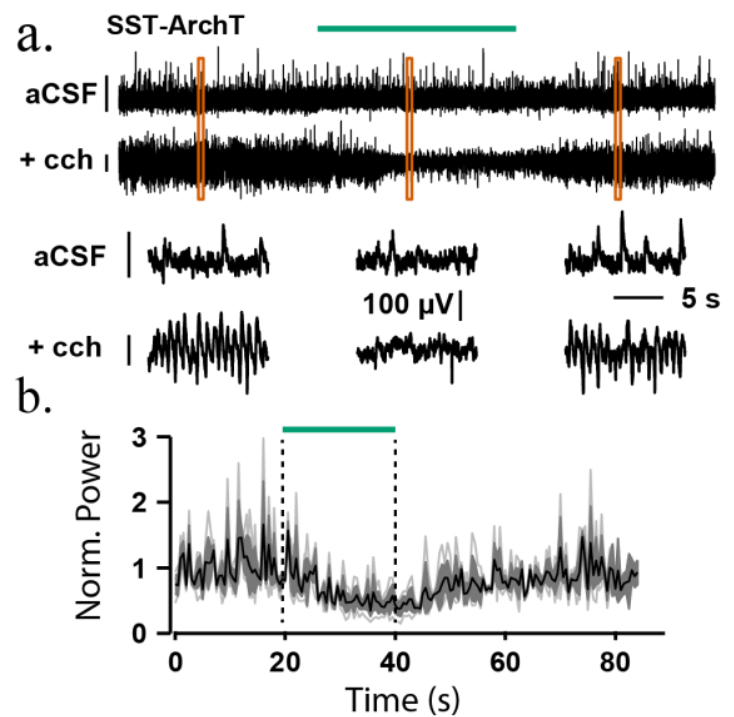

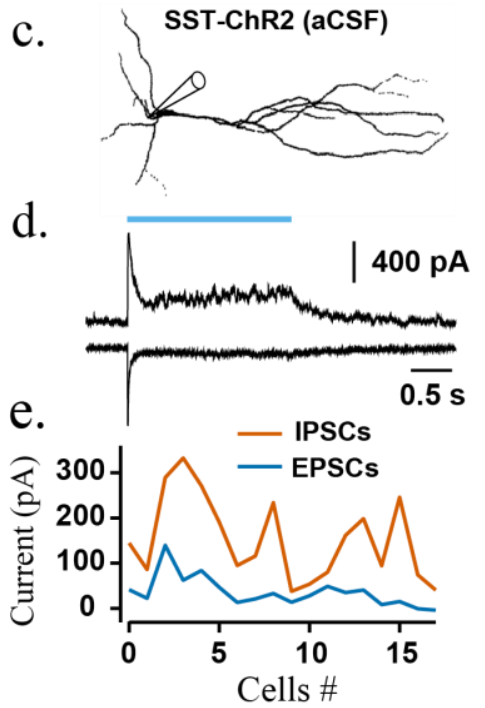

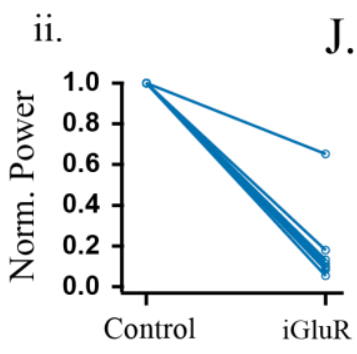

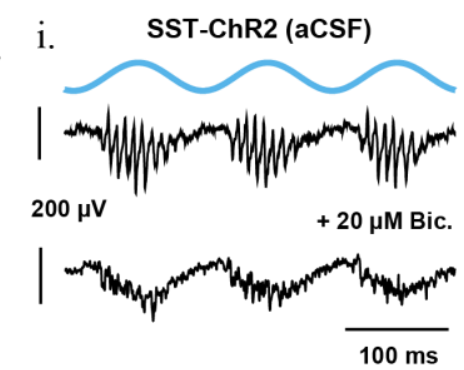

f.

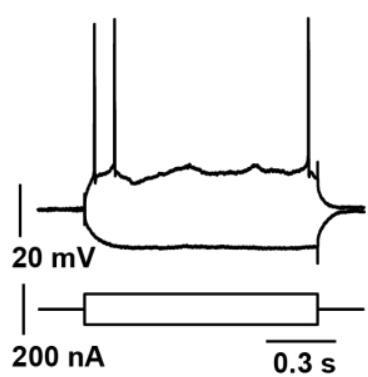

g.
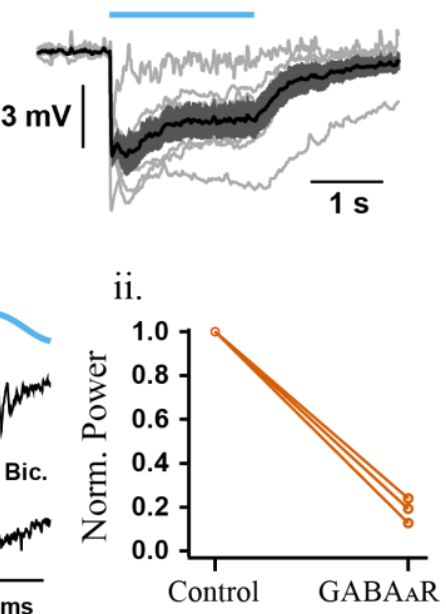

Supplementary Figure 5: (a-b) Responses to laser illumination in SST-cre mice expressing ArchT-GFP. a) Representative LFP recording with and without the presence of Cch during green laser illumination (approx. $18.6 \mathrm{~mW}$ ). Orange squares represent the bottom sections of LFP that were magnified. $\mathrm{b}$ ) Change in power-area normalised to baseline calculated in 1 second bins across experiments $(n=3)$ in the absence of Cch; Dotted lines indicate the duration of laser illumination. (c-e) Voltage clamp recordings from putative pyramidal cells during photo-activation of SST+ interneurons $(1.53 \mathrm{~mW})$. c) Reconstruction of a recorded cell with typical pyramidal cell morphology in CA3 hippocampal area. d) Representative voltage clamp recording of the cell in c) held at $0 \mathrm{mV}$ (top) and $-70 \mathrm{mV}$ (bottom) to isolate IPSCs and EPSCs, respectively. e) Comparison of EPSCs and IPSCs during SST+ interneuron photo-activation across cells $(n=18)$. Blue line $=$ EPSCs, Orange line $=$ IPSCs. $(\mathrm{f}-\mathrm{g})$ Perforated patch recordings in CA3 pyramidal cell layer in SST cre mice expressing ChR2-mcherry. f) Current clamp recording in a putative pyramidal cell in response to depolarising and hyperpolarising current injections. g) Hyperpolarisation of the membrane voltage of perforated patched cells $(\mathrm{n}=6)$ during blue light illumination $(1.53 \mathrm{~mW})$. Grey traces represent individual cells, black trace the average and dark grey shaded area the SEM. (h-j) pharmacology of de-novo oscillations induced by sinusoidal blue light illumination in SST-cre mice expressing ChR2-mcherry $(1-10 \mathrm{~mW})$. Representative LFP recording in CA3 before (top) and after (bottom) application of h.i) iGLUR blockers and j.i) $\mathrm{GABA}_{A} \mathrm{R}$ blockers. Power-area change before (control) and after application h.ii) iGLUR blockers and j.ii) $\mathrm{GABA}_{\mathrm{A}} \mathrm{R}$ blockers. GluR blockers used: $20 \mu \mathrm{M}$ CNQX, $40 \mu \mathrm{M}$ AP5, $\mathrm{n}=3 ; 10 \mu \mathrm{M}$ CNQX, $20 \mu \mathrm{M}$ $\mathrm{AP5}, \mathrm{n}=1 ; 20 \mu \mathrm{M}$ CNQX, $\mathrm{n}=1 ; 3 \mathrm{mM}$ kynurenic, $\mathrm{n}=3$. GABA $\mathrm{A}$ blockers: $20 \mu \mathrm{M}$ Bicuculline, $\mathrm{n}=2 ; 20$ $\mu \mathrm{M}$ Gabazine, $\mathrm{n}=1$. 


\section{Discussion}

Gamma oscillations depend on synchronised synaptic inhibition, and there is a wealth of evidence suggesting that perisomatic-targeting PV+ interneurons provide the critical inhibitory output for both current and rhythm generation (Mann et al., 2005; Bartos, Vida \& Jonas, 2007; Oren, Hájos \& Paulsen, 2010; Tukker et al., 2013; Cardin, 2016; Sohal, 2016; Penttonen et al., 1998b). Here, we used optogenetic manipulation of PV+ and SST+ interneurons to explore whether PV+ interneurons have a selective role in gamma rhythmogenesis in the hippocampal CA3 ex vivo. Our findings suggest that optogenetically disrupting interneuronal activity, via either photo-inhibition or photoexcitation, generally leads to a decrease in the power and increase in the frequency of ongoing cholinergically-induced slow gamma oscillations. This suggests that both PV+ and SST+ interneurons play key roles in maintaining slow gamma oscillations, and the key differences were that (i) cholinergically-induced gamma oscillations were more readily disrupted by photo-inhibition of SST+ interneurons rather than PV+ interneurons, (ii) manipulation of SST+ interneurons modulated gamma oscillation frequency more robustly than that of PV+ interneurons, and (iii) photostimulation of SST+ interneurons could also induce de novo fast gamma oscillations.

Slow gamma oscillations in the hippocampal CA3 appear to be generated by synaptic feedback loops between excitatory pyramidal neurons and perisomatic-targeting interneurons, both in brain slices (Fisahn et al., 1998; Hajos, 2004; Mann et al., 2005; Oren et al., 2006; Butler, Hay \& Paulsen, 2018) and in vivo (Bragin et al., 1995; Csicsvari et al., 2003; Fuchs et al., 2007). In such feedback loops, the period of the oscillation largely reflects the effective time course of inhibitory postsynaptic potentials in the pyramidal cells, which should become shorter with smaller compound inhibitory synaptic currents and/or increased pyramidal cell excitability. The amplitude of the oscillation recorded in the LFP also reflects the amplitude of phasic inhibitory currents in pyramidal neurons (Mann et al., 2005; Oren, Hájos \& Paulsen, 2010), and during spontaneous gamma oscillations there 
is a strong correlation between the instantaneous period and amplitude of each gamma cycle

368 (Atallah \& Scanziani, 2009). One might thus expect disinhibition to decrease the amplitude and 369 increase the frequency of gamma oscillations, which is largely what we observed with photo370 inhibition of either PV+ or SST+ interneurons. Observing similar effects with photo-stimulation of 371 interneurons might be somewhat more surprising. However, our interpretation is that this also effectively disrupts synchronisation within synaptic feedback loops, by silencing a subpopulation of pyramidal cells and thus 'knocking out' part of the gamma oscillating network. In both types of optogenetic manipulation, we could thus be recording the activity in residual parts of the network that can maintain synaptic feedback loops, albeit with weaker synchronised inhibition.

While photoinhibition of either PV+ or SST+ interneurons was able to disrupt cholinergically-induced gamma oscillations, it was necessary to use high-powered laser illumination of PV+ interneurons to consistently reduce gamma power, and the oscillations were not abolished under our stimulation paradigms. This is not inconsistent with PV+ interneurons playing a key role in the synaptic feedback loops generating gamma oscillations in the hippocampal CA3, as such a microcircuit should resist disinhibition. Indeed, strong laser illumination was necessary to biochemically silence PV+ interneuron terminals (El-Gaby et al., 2016), and thus break this feedback loop. The lack of consistent effects on the frequency of cholinergically-induced gamma oscillations may also be due to the difficulty in silencing PV+ interneurons. Alternatively, it is possible that the remaining PV+ interneurons take longer to fire in each gamma cycle due to the Arch-induced hyperpolarisation. Combined with a more excitable pyramidal neurons, which recover more rapidly form synaptic inhibition, this could leave the overall oscillation frequency unchanged.

It was recently suggested that SST+ interneurons, but not PV+ interneurons, contribute to the 
hippocampal gamma oscillations, but are consistent with an important role for SST+ interneurons

in gamma rhythmogenesis across cortical circuits. However, SST+ interneurons largely target the dendritic domains of pyramidal cells, and thus it remains difficult to see how they could directly contribute to the precise timing of pyramidal cell spiking during fast brain oscillations, such as cholinergically-induced gamma oscillations in hippocampal CA3. SST+ bistratified interneurons have been proposed to have similar properties to fast spiking PV+ interneurons, and also form a small portion of synapses close to the soma (Somogyi \& Klausberger, 2005; Muller \& Remy, 2014), but have been reported to exhibit decreased GABA release under cholinergic stimulation (Gulyás et al., 2010). It therefore seems likely that the importance of SST+ interneurons to the generation of cholinergically-induced hippocampal gamma oscillations lies in their modulation of perisomatic feedback loops, via effects on both pyramidal neuron excitability, and the spike rate and precision in PV+ interneurons (Savanthrapadian et al., 2014).

While optogenetic manipulation of SST+ interneurons consistently disrupted slow gamma oscillations, we found that photo-stimulation of SST+ interneurons could also induce de novo fast gamma oscillations. These GABAergic interneurons should provide a powerful source of circuit inhibition (Somogyi \& Klausberger, 2005; Pfeffer et al., 2013; Taniguchi et al., 2011; Leão et al., 2012; sustained photo-stimulation of SST+ interneurons did not significantly inhibit the activity of STTneurons, and that pulsed stimulation could drive network excitation. It may be that the hyperactivation of a dense plexus of SST+ processes in the dendritic layers leads to bystander effects on nearby SST- neurons, possibly via ephaptic interactions and/or changes in the extracellular ionic environment (Anastassiou et al., 2011; Ferenczi et al., 2016b), which counteracts the effects of synaptic inhibition. The generation of fast gamma oscillations appeared to depend on the maintenance of network excitability, as the oscillations were attenuated by block of iGluR. However, the spiking of the majority of STT- RS neurons was only weakly coupled to the phase of light-induced 
416 fast gamma oscillations, and without a consistent population spike phase preference, while light417 sensitive putative SST+ interneurons showed reliable phase-locking. This could be consistent with 418 fast gamma oscillations representing rhythmic dendritic inhibition from STT+ interneurons, with 419 only weak effects on the spike rate and timing of other neurons in the network.

420 The mechanism by which a network of SST+ interneurons might generate fast gamma oscillations 421 remains obscure. In neocortex, SST+ interneurons avoid inhibiting each other (Pfeffer et al., 2013), 422 although there is evidence for sparse synaptic interactions between SST+ interneurons in the 423 hippocampus (Savanthrapadian et al., 2014), and for more generic coupling via gap junctions (Baude 424 et al., 2007). More experiments are required to resolve the mechanisms by which optogenetic 425 manipulation of interneurons influences hippocampal gamma oscillations, and whether STT+ 426 neurons contribute to fast hippocampal gamma oscillations during theta and non-theta states in 427 vivo (Sullivan et al., 2011). However, our findings suggest that SST+ interneurons exert powerful 428 control over the power and frequency of slow hippocampal gamma oscillations, and can switch the 429 network between slow and fast gamma states. 


\section{REFERENCES}

Akam, T. \& Kullmann, D.M. (2010) Oscillations and Filtering Networks Support Flexible Routing of Information. Neuron. [Online] 67 (2), 308-320. Available from: doi:10.1016/j.neuron.2010.06.019.

Anastassiou, C.A., Perin, R., Markram, H. \& Koch, C. (2011) Ephaptic coupling of cortical neurons. Nature Neuroscience. [Online] 14 (2), 217-223. Available from: doi:10.1038/nn.2727.

Andersen, P., Bliss, T.V.P. \& Skrede, K.K. (1971) Unit analysis of hippocampal population spikes. Experimental Brain Research. [Online] 13 (2), 208-221. Available from: doi:10.1007/BF00234086.

Atallah, B. V. \& Scanziani, M. (2009) Instantaneous Modulation of Gamma Oscillation Frequency by Balancing Excitation with Inhibition. Neuron. [Online] 62 (4), 566-577. Available from: doi:10.1016/J.NEURON.2009.04.027.

Bartos, M. \& Elgueta, C. (2012) Functional characteristics of parvalbumin- and cholecystokininexpressing basket cells. The Journal of Physiology. [Online] 590 (4), 669-681. Available from: doi:10.1113/jphysiol.2011.226175.

Bartos, M., Vida, I. \& Jonas, P. (2007) Synaptic mechanisms of synchronized gamma oscillations in inhibitory interneuron networks. Nature reviews. Neuroscience. [Online] 8 (1), 45-56. Available from: doi:10.1038/nrn2044.

Basar-Eroglu, C., Brand, A., Hildebrandt, H., Karolina Kedzior, K., et al. (2007) Working memory related gamma oscillations in schizophrenia patients. International Journal of Psychophysiology. [Online] 64 (1), 39-45. Available from: doi:10.1016/j.ijpsycho.2006.07.007. 
delays. Current Opinion in Neurobiology. [Online] 31, 173-180. Available from: doi:10.1016/J.CONB.2014.11.001.

Baude, A., Bleasdale, C., Dalezios, Y., Somogyi, P., et al. (2007) Immunoreactivity for the GABAA Receptor 1 Subunit, Somatostatin and Connexin36 Distinguishes Axoaxonic, Basket, and Bistratified Interneurons of the Rat Hippocampus. Cerebral Cortex. [Online] 17 (9), 20942107. Available from: doi:10.1093/cercor/bhl117.

Beierlein, M., Gibson, J.R. \& Connors, B.W. (2000) A network of electrically coupled interneurons drives synchronized inhibitionin neocortex. Nature Neuroscience. [Online] 3 (9), 904-910. Available from: doi:10.1038/78809.

Betterton, R.T., Broad, L.M., Tsaneva-Atanasova, K. \& Mellor, J.R. (2017) Acetylcholine modulates gamma frequency oscillations in the hippocampus by activation of muscarinic M1 receptors Panayiota Poirazi (ed.). European Journal of Neuroscience. [Online] 45 (12), 1570-1585. Available from: doi:10.1111/ejn.13582.

Boyden, E.S., Zhang, F., Bamberg, E., Nagel, G., et al. (2005) Millisecond-timescale, genetically targeted optical control of neural activity. Nature Neuroscience. [Online] 8 (9), 1263-1268. Available from: doi:10.1038/nn1525.

Bragin, A., Jandó, G., Nádasdy, Z., Hetke, J., et al. (1995) Gamma (40-100 Hz) oscillation in the hippocampus of the behaving rat. The Journal of neuroscience : the official journal of the Society for Neuroscience. [Online] 15 (1 Pt 1), 47-60. Available from: doi:https://doi.org/10.1523/JNEUROSCI.15-01-00047.1995.

Burns, S.P., Xing, D. \& Shapley, R.M. (2011) Is gamma-band activity in the local field potential of V1 cortex a 'clock' or filtered noise? The Journal of neuroscience : the official journal of the Society for Neuroscience. [Online] 31 (26), 9658-9664. Available from: 
doi:10.1523/JNEUROSCI.0660-11.2011.

477

478

479

480

481

482

483

484

485

486

487

488

489

490

491

492

493

494

495

496

497

Butler, J.L., Hay, Y.A. \& Paulsen, O. (2018) Comparison of three gamma oscillations in the mouse entorhinal-hippocampal system. European Journal of Neuroscience. [Online] Available from: doi:10.1111/ejn.13831.

Butler, J.L., Mendonça, P.R.. F., Robinson, H.P.C. \& Paulsen, O. (2016) Intrinsic Cornu Ammonis Area 1 Theta-Nested Gamma Oscillations Induced by Optogenetic Theta Frequency Stimulation. The Journal of neuroscience : the official journal of the Society for Neuroscience. [Online] 36 (15), 4155-4169. Available from: doi:10.1523/JNEUROSCI.3150-15.2016.

Butler, J.L. \& Paulsen, O. (2014) The Hippocampal Cacophony: Multiple Layers of Communication. Neuron. [Online] 84 (2), 251-253. Available from: doi:10.1016/J.NEURON.2014.10.017.

Buzsáki, G. \& Wang, X.-J. (2012) Mechanisms of Gamma Oscillations. Annual Review of Neuroscience. [Online] 35 (1), 203-225. Available from: doi:10.1146/annurev-neuro-062111150444.

Cardin, J.A. (2016) Snapshots of the Brain in Action: Local Circuit Operations through the Lens of $Y$ Oscillations. Journal of Neuroscience. [Online] 36 (41), 10496-10504. Available from: doi:10.1523/JNEUROSCI.1021-16.2016.

Cardin, J.A., Carlén, M., Meletis, K., Knoblich, U., et al. (2009) Driving fast-spiking cells induces gamma rhythm and controls sensory responses. Nature. [Online] 459 (7247), 663-667. Available from: doi:10.1038/nature08002.

Chen, G., Zhang, Y., Li, X., Zhao, X., et al. (2017) Distinct Inhibitory Circuits Orchestrate Cortical beta and gamma Band Oscillations. Neuron. [Online] 96 (6), 1403-1418.e6. Available from: doi:10.1016/j.neuron.2017.11.033.

Chow, B.Y., Han, X., Dobry, A.S., Qian, X., et al. (2010) High-performance genetically targetable 
optical neural silencing by light-driven proton pumps. Nature. [Online] 463 (7277), 98-102. Available from: doi:10.1038/nature08652.

Colgin, L.L., Denninger, T., Fyhn, M., Hafting, T., et al. (2009) Frequency of gamma oscillations routes flow of information in the hippocampus. Nature. [Online] 462 (7271), 353-357. Available from: doi:10.1038/nature08573.

Craig, M.T. \& McBain, C.J. (2015) Fast gamma oscillations are generated intrinsically in CA1 without the involvement of fast-spiking basket cells. The Journal of neuroscience : the official journal of the Society for Neuroscience. [Online] 35 (8), 3616-3624. Available from: doi:10.1523/JNEUROSCI.4166-14.2015.

Csicsvari, J., Jamieson, B., Wise, K.D. \& Buzsáki, G. (2003) Mechanisms of gamma oscillations in the hippocampus of the behaving rat. Neuron. [Online] 37 (2), 311-322. Available from: doi:10.1016/S0896-6273(02)01169-8.

El-Gaby, M., Zhang, Y., Wolf, K., Schwiening, C.J., et al. (2016) Archaerhodopsin Selectively and Reversibly Silences Synaptic Transmission through Altered pH. Cell Reports. [Online] 16 (8), 2259-2268. Available from: doi:10.1016/J.CELREP.2016.07.057.

Fee, M.S., Mitra, P.P. \& Kleinfeld, D. (1996) Automatic sorting of multiple unit neuronal signals in the presence of anisotropic and non-Gaussian variability. Journal of Neuroscience Methods. [Online] 69 (2), 175-188. Available from: doi:10.1016/S0165-0270(96)00050-7.

Ferenczi, E.A., Vierock, J., Atsuta-Tsunoda, K., Tsunoda, S.P., et al. (2016a) Optogenetic approaches addressing extracellular modulation of neural excitability. Scientific Reports. [Online] 6 (1), 23947. Available from: doi:10.1038/srep23947.

Ferenczi, E.A., Vierock, J., Atsuta-Tsunoda, K., Tsunoda, S.P., et al. (2016b) Optogenetic approaches addressing extracellular modulation of neural excitability. Scientific Reports. [Online] 6 (1), 
23947. Available from: doi:10.1038/srep23947.

523

524

525

526

527

528

529

530

531

532

533

534

535

536

537

538

539

540

541

542

543

544

Fisahn, A., Pike, F.G., Buhl, E.. H. \& Paulsen, O. (1998) Cholinergic induction of network oscillations at $40 \mathrm{~Hz}$ in the hippocampus in vitro. Nature. [Online] 394 (6689), 186-189. Available from: doi:10.1038/28179.

Fries, P. (2005) A mechanism for cognitive dynamics: neuronal communication through neuronal coherence. Trends in Cognitive Sciences. [Online] 9 (10), 474-480. Available from: doi:10.1016/J.TICS.2005.08.011.

Fries, P. (2015) Rhythms for Cognition: Communication through Coherence. Neuron. [Online] 88 (1), 220-235. Available from: doi:10.1016/j.neuron.2015.09.034.

Fries, P., Reynolds, J.H., Rorie, A.E. \& Desimone, R. (2001) Modulation of oscillatory neuronal synchronization by selective visual attention. Science (New York, N.Y.). [Online] 291 (5508), 1560-1563. Available from: doi:10.1126/science.291.5508.1560.

Fuchs, E.C., Zivkovic, A.R., Cunningham, M.O., Middleton, S., et al. (2007) Recruitment of Parvalbumin-Positive Interneurons Determines Hippocampal Function and Associated Behavior. Neuron. [Online] 53 (4), 591-604. Available from: doi:10.1016/J.NEURON.2007.01.031.

Gloveli, T., Dugladze, T., Saha, S., Monyer, H., et al. (2005) Differential involvement of oriens/pyramidale interneurones in hippocampal network oscillations In vitro. The Journal of Physiology. [Online] 562 (1), 131-147. Available from: doi:10.1113/jphysiol.2004.073007.

Gulyás, A.I., Szabó, G.G., Ulbert, I., Holderith, N., et al. (2010) Parvalbumin-containing fast-spiking basket cells generate the field potential oscillations induced by cholinergic receptor activation in the hippocampus. The Journal of neuroscience : the official journal of the Society for Neuroscience. [Online] 30 (45), 15134-15145. Available from: doi:10.1523/JNEUROSCI.4104- 
10.2010.

546

547

548

549

550

551

552

553

554

555

556

557

558

559

560

561

562

563

564

565

566

Hajos, N. (2004) Spike Timing of Distinct Types of GABAergic Interneuron during Hippocampal Gamma Oscillations In Vitro. Journal of Neuroscience. [Online] 24 (41), 9127-9137. Available from: doi:10.1523/JNEUROSCI.2113-04.2004.

Hájos, N. \& Paulsen, O. (2009) Network mechanisms of gamma oscillations in the CA3 region of the hippocampus. Neural Networks. [Online] 22 (8), 1113-1119. Available from: doi:10.1016/j.neunet.2009.07.024.

Hakim, R., Shamardani, K. \& Adesnik, H. (2018) A neural circuit for gamma-band coherence across the retinotopic map in mouse visual cortex. eLife. [Online] 7, e28569. Available from: doi:10.7554/eLife.28569.

Hasenstaub, A., Shu, Y., Haider, B., Kraushaar, U., et al. (2005) Inhibitory Postsynaptic Potentials Carry Synchronized Frequency Information in Active Cortical Networks. Neuron. [Online] 47 (3), 423-435. Available from: doi:10.1016/J.NEURON.2005.06.016.

Herman, A.M., Huang, L., Murphey, D.K., Garcia, I., et al. (2014) Cell type-specific and timedependent light exposure contribute to silencing in neurons expressing Channelrhodopsin-2. eLife. [Online] 3, e01481. Available from: doi:10.7554/eLife.01481.

Herrmann, C.S. \& Demiralp, T. (2005) Human EEG gamma oscillations in neuropsychiatric disorders. Clinical Neurophysiology. [Online] 116 (12), 2719-2733. Available from: doi:10.1016/J.CLINPH.2005.07.007.

Hofer, S.B., Ko, H., Pichler, B., Vogelstein, J., et al. (2011) Differential connectivity and response dynamics of excitatory and inhibitory neurons in visual cortex. Nature Neuroscience. [Online] 14 (8), 1045-1052. Available from: doi:10.1038/nn.2876.

Hu, H., Gan, J. \& Jonas, P. (2014) Interneurons. Fast-spiking, parvalbumin+ GABAergic 
interneurons: from cellular design to microcircuit function. Science (New York, N.Y.). [Online] 345 (6196), 1255263. Available from: doi:10.1126/science.1255263.

Kim, D., Jeong, H., Lee, J., Ghim, J.-W., et al. (2016) Distinct Roles of Parvalbumin- and Somatostatin-Expressing Interneurons in Working Memory. Neuron. [Online] 92 (4), 902-915. Available from: doi:10.1016/J.NEURON.2016.09.023.

Kohus, Z., Káli, S., Rovira-Esteban, L., Schlingloff, D., et al. (2016) Properties and dynamics of inhibitory synaptic communication within the CA3 microcircuits of pyramidal cells and interneurons expressing parvalbumin or cholecystokinin. The Journal of Physiology. [Online] 594 (13), 3745-3774. Available from: doi:10.1113/JP272231.

Lasztóczi, B. \& Klausberger, T. (2016) Hippocampal Place Cells Couple to Three Different Gamma Oscillations during Place Field Traversal. Neuron. [Online] 91 (1), 34-40. Available from: doi:10.1016/J.NEURON.2016.05.036.

Leão, R.N., Mikulovic, S., Leão, K.E., Munguba, H., et al. (2012) OLM interneurons differentially modulate CA3 and entorhinal inputs to hippocampal CA1 neurons. Nature Neuroscience. [Online] 15 (11), 1524-1530. Available from: doi:10.1038/nn.3235.

Lovett-Barron, M., Kaifosh, P., Kheirbek, M.A., Danielson, N., et al. (2014) Dendritic inhibition in the hippocampus supports fear learning. Science (New York, N.Y.). [Online] 343 (6173), 857863. Available from: doi:10.1126/science.1247485.

Lovett-Barron, M., Turi, G.F., Kaifosh, P., Lee, P.H., et al. (2012) Regulation of neuronal input transformations by tunable dendritic inhibition. Nature Neuroscience. [Online] 15 (3), 423430. Available from: doi:10.1038/nn.3024.

Ma, Y., Hu, H., Berrebi, A.S., Mathers, P.H., et al. (2006) Distinct subtypes of somatostatincontaining neocortical interneurons revealed in transgenic mice. The Journal of neuroscience: 

the official journal of the Society for Neuroscience. [Online] 26 (19), 5069-5082. Available from: doi:10.1523/JNEUROSCI.0661-06.2006.

Mann, E.O., Radcliffe, C.A. \& Paulsen, O. (2005) Hippocampal gamma-frequency oscillations: from interneurones to pyramidal cells, and back. The Journal of Physiology. [Online] 562 (1), 55-63. Available from: doi:10.1113/jphysiol.2004.078758.

Mann, E.O., Suckling, J.M., Hajos, N., Greenfield, S.A., et al. (2005) Perisomatic Feedback Inhibition Underlies Cholinergically Induced Fast Network Oscillations in the Rat Hippocampus In Vitro. Neuron. [Online] 45 (1), 105-117. Available from: doi:10.1016/j.neuron.2004.12.016.

Muller, C. \& Remy, S. (2014) Dendritic inhibition mediated by O-LM and bistratified interneurons in the hippocampus. Frontiers in Synaptic Neuroscience. [Online] 6, 23. Available from: doi:10.3389/fnsyn.2014.00023.

Nagel, G., Szellas, T., Huhn, W., Kateriya, S., et al. (2003) Channelrhodopsin-2, a directly light-gated cation-selective membrane channel. Proceedings of the National Academy of Sciences of the United States of America. [Online] 100 (24), 13940-13945. Available from: doi:10.1073/pnas.1936192100.

Oren, I., Hájos, N. \& Paulsen, O. (2010) Identification of the current generator underlying cholinergically induced gamma frequency field potential oscillations in the hippocampal CA3 region. The Journal of Physiology. [Online] 588 (5), 785-797. Available from: doi:10.1113/jphysiol.2009.180851.

Oren, I., Mann, E.O., Paulsen, O. \& Hájos, N. (2006) Synaptic currents in anatomically identified CA3 neurons during hippocampal gamma oscillations in vitro. The Journal of neuroscience: the official journal of the Society for Neuroscience. [Online] 26 (39), 9923-9934. Available from: doi:10.1523/JNEUROSCI.1580-06.2006. 
Packer, A.M. \& Yuste, R. (2011) Dense, unspecific connectivity of neocortical parvalbumin-positive interneurons: a canonical microcircuit for inhibition? The Journal of neuroscience : the official journal of the Society for Neuroscience. [Online] 31 (37), 13260-13271. Available from: doi:10.1523/JNEUROSCI.3131-11.2011.

Penttonen, M., Kamondi, A., Acsady, L. \& Buzsaki, G. (1998a) Gamma frequency oscillation in the hippocampus of the rat: intracellular analysis in vivo. European Journal of Neuroscience. [Online] 10 (2), 718-728. Available from: doi:10.1046/j.1460-9568.1998.00096.x.

Penttonen, M., Kamondi, A., Acsady, L. \& Buzsaki, G. (1998b) Gamma frequency oscillation in the hippocampus of the rat: intracellular analysis in vivo. European Journal of Neuroscience. [Online] 10 (2), 718-728. Available from: doi:10.1046/j.1460-9568.1998.00096.x.

Pfeffer, C.K., Xue, M., He, M., Huang, Z.J., et al. (2013) Inhibition of inhibition in visual cortex: the logic of connections between molecularly distinct interneurons. Nature Neuroscience. [Online] 16 (8), 1068-1076. Available from: doi:10.1038/nn.3446.

Pike, F.G., Goddard, R.S., Suckling, J.M., Ganter, P., et al. (2000) Distinct frequency preferences of different types of rat hippocampal neurones in response to oscillatory input currents. The Journal of Physiology. [Online] 529 (1), 205-213. Available from: doi:10.1111/j.14697793.2000.00205.x.

Pouille, F. \& Scanziani, M. (2001) Enforcement of temporal fidelity in pyramidal cells by somatic feed-forward inhibition. Science (New York, N.Y.). [Online] 293 (5532), 1159-1163. Available from: doi:10.1126/science.1060342.

Quian Quiroga, R. (2009) What is the real shape of extracellular spikes? Journal of Neuroscience Methods. [Online] 177 (1), 194-198. Available from: doi:10.1016/J.JNEUMETH.2008.09.033. 
Wavelets and Superparamagnetic Clustering. Neural Computation. [Online] 16 (8), 16611687. Available from: doi:10.1162/089976604774201631.

Ray, S. \& Maunsell, J.H.R. (2015) Do gamma oscillations play a role in cerebral cortex? Trends in Cognitive Sciences. [Online] 19 (2), 78-85. Available from: doi:10.1016/J.TICS.2014.12.002.

Royer, S. eacute bastien, Zemelman, B. V, Losonczy, A., Kim, J., et al. (2012) Control of timing, rate and bursts of hippocampal place cells by dendritic and somatic inhibition. Nature Neuroscience. [Online] 15 (5), 1-10. Available from: doi:10.1038/nn.3077.

Savanthrapadian, S., Meyer, T., Elgueta, C., Booker, S.A., et al. (2014) Synaptic Properties of SOMand CCK-Expressing Cells in Dentate Gyrus Interneuron Networks. Journal of Neuroscience. [Online] 34 (24), 8197-8209. Available from: doi:10.1523/JNEUROSCI.5433-13.2014.

Schomburg, E.W., Fernández-Ruiz, A., Mizuseki, K., Berényi, A., et al. (2014) Theta phase segregation of input-specific gamma patterns in entorhinal-hippocampal networks. Neuron. [Online] 84 (2), 470-485. Available from: doi:10.1016/j.neuron.2014.08.051.

Sohal, V.S. (2016) How Close Are We to Understanding What (if Anything) Oscillations Do in Cortical Circuits? Journal of Neuroscience. [Online] 36 (41), 10489-10495. Available from: doi:10.1523/JNEUROSCI.0990-16.2016.

Sohal, V.S., Zhang, F., Yizhar, O. \& Deisseroth, K. (2009) Parvalbumin neurons and gamma rhythms enhance cortical circuit performance. Nature. [Online] 459 (7247), 698-702. Available from: doi:10.1038/nature07991.

Somogyi, P. \& Klausberger, T. (2005) Defined types of cortical interneurone structure space and spike timing in the hippocampus. The Journal of physiology. [Online] 562 (Pt 1), 9-26. Available from: doi:10.1113/jphysiol.2004.078915. 
spatial encoding in working memory. Nature. [Online] 522 (7556), 309-314. Available from: doi:10.1038/nature14445.

Sullivan, D., Csicsvari, J., Mizuseki, K., Montgomery, S., et al. (2011) Relationships between hippocampal sharp waves, ripples, and fast gamma oscillation: influence of dentate and entorhinal cortical activity. The Journal of neuroscience : the official journal of the Society for Neuroscience. [Online] 31 (23), 8605-8616. Available from: doi:10.1523/JNEUROSCI.029411.2011.

Taniguchi, H., He, M., Wu, P., Kim, S., et al. (2011) A Resource of Cre Driver Lines for Genetic Targeting of GABAergic Neurons in Cerebral Cortex. Neuron. [Online] 71 (6), 995-1013. Available from: doi:10.1016/J.NEURON.2011.07.026.

Tukker, J.J., Fuentealba, P., Hartwich, K., Somogyi, P., et al. (2007) Cell Type-Specific Tuning of Hippocampal InterneuronFiring during Gamma Oscillations. [Online] 27 (31), 1-6. Available from: doi:10.1523/JNEUROSCI.1685-07.2007.

Tukker, J.J., Lasztóczi, B., Katona, L., Roberts, J.D.B., et al. (2013) Distinct dendritic arborization and in vivo firing patterns of parvalbumin-expressing basket cells in the hippocampal area CA3. The Journal of neuroscience : the official journal of the Society for Neuroscience. [Online] 33 (16), 6809-6825. Available from: doi:10.1523/JNEUROSCI.5052-12.2013.

Uhlhaas, P.J. \& Singer, W. (2010) Abnormal neural oscillations and synchrony in schizophrenia. Nature Reviews Neuroscience. [Online] 11 (2), 100-113. Available from: doi:10.1038/nrn2774.

Uhlhaas, P.J. \& Singer, W. (2006) Neural Synchrony in Brain Disorders: Relevance for Cognitive Dysfunctions and Pathophysiology. Neuron. [Online] 52 (1), 155-168. Available from: doi:10.1016/j.neuron.2006.09.020.

Urban-Ciecko, J. \& Barth, A.L. (2016) Somatostatin-expressing neurons in cortical networks. Nature 
Reviews Neuroscience. [Online] 17 (7), 401-409. Available from: doi:10.1038/nrn.2016.53.

684

685

686

687

688

689

690

691

692

693

694

695

696

697

698

699

700

701

702

Veit, J., Hakim, R., Jadi, M.P., Sejnowski, T.J., et al. (2017) Cortical gamma band synchronization through somatostatin interneurons. Nature Neuroscience. [Online] 20 (7), 951-959. Available from: doi:10.1038/nn.4562.

Whittington, M.A., Traub, R.D. \& Jefferys, J.G.R. (1995) Synchronized oscillations in interneuron networks driven by metabotropic glutamate receptor activation. Nature. [Online] 373 (6515), 612-615. Available from: doi:10.1038/373612a0.

Wierenga, C.. \& Wadman, W.. (2003) Functional relation between interneuron input and population activity in the rat hippocampal cornu ammonis 1 area. Neuroscience. [Online] 118 (4), 1129-1139. Available from: doi:10.1016/S0306-4522(03)00060-5.

Womelsdorf, T. \& Everling, S. (2015) Long-Range Attention Networks: Circuit Motifs Underlying Endogenously Controlled Stimulus Selection. Trends in Neurosciences. [Online] 38 (11), 682700. Available from: doi:10.1016/J.TINS.2015.08.009.

Yamamoto, J., Suh, J., Takeuchi, D. \& Tonegawa, S. (2014) Successful Execution of Working Memory Linked to Synchronized High-Frequency Gamma Oscillations. Cell. [Online] 157 (4), 845-857. Available from: doi:10.1016/J.CELL.2014.04.009.

Zar, J.H. (1999) Biostatistical analysis. In: Prentice Hall. 4th edition. Englewood Cliffs, New Jersey. p. 929.

\section{Materials and Methods}

\section{Transgenic mice}

All procedures were performed according to the United Kingdom Animals Scientific Procedures Act (ASPA) 1986 and the University of Oxford guidelines. Adult (older than 8 weeks, both male and 
female) PV-cre (B6;129P2-Pvalbtm1(cre)Arbr/J), PV-cre-Ai9 (PV-Cre x Gt ROSA (CAG-tdTomato) Hze/J), and SST-cre mice (Sst tm2.1(cre)Zjh/J) were used for all experiments.

\section{Stereotaxic viral injections}

Anaesthesia was induced in mice with $4 \%$ isoflurane/medical oxygen mixture ( $2 \mathrm{~L}$ per min). The area around the head was shaved and cleaned in preparation for scalp incision. Anaesthesia was subsequently maintained using $1.5-2.5 \%$ isoflurane at a rate of $2 \mathrm{~L}$ per $\min$. Before the onset of the procedure a cocktail of systemic peri-operative analgesics (Metacam $1 \mathrm{mg} / \mathrm{Kg}$ and Vetergesic $0.1 \mathrm{mg} / \mathrm{Kg}$ ) and a local analgesic (Marcaine 10mg/Kg) were administered subcutaneously (Oxford University Veterinary Services). Following, antibiotic solution was applied on the head and an incision of the scalp was performed that allowed a small craniotomy to be made. A 33/34-gauge needle was attached on a Hamilton Microliter Syringe and used to inject the virus solution at a rate of $\approx 100 \mathrm{~nL} / \mathrm{min}$ (viral concentration $\approx 10^{12}$ genome copies per $\mathrm{mL}$ ). After every injection, the needle was left stationary for at least three minutes to allow diffusion of virus in the surrounding area. The virus solution was injected with the aid of a stereotaxic frame into ventral CA3 area of hippocampus ( $2.7 \mathrm{~mm}$ caudal and $2.75 \mathrm{~mm}$ lateral from Bregma). A total of $600-800 \mathrm{~nL}$ were injected at two depths (300 - $400 \mathrm{~nL}$ at $3.1 \mathrm{~mm}$ and $300-400 \mathrm{~nL}$ at $2.7 \mathrm{~mm}$ ). Following the injection, local analgesic (Marcaine $10 \mathrm{mg} / \mathrm{Kg}$ ) was applied on the incised scalp before it was sutured. The animals were then transferred in a heating chamber and allowed to recover. The animals were monitored, and welfare scored in the following days to ensure that they properly recovered after surgery. Injected mice were assessed for viral expression after a minimum of 3 weeks. All viral constructs were acquired from Vector Core Facilities, Gene Therapy Centre (North Carolina, UNC). Viral constructs used: AAV5-EF1a-DIO-ChR2-mCherry, AAV5-EF1a-DIO-ChR2-eYFP, AAV-EF1a-DIO-Arch3.0-EYFP, AAVEf1a-DIO-hChR2(E123T-T159C)-p2A-mCherry-WPRE (Dr. Karl Deisseroth), and AAV-CAG-FLEXArchT-GFP (Dr. Ed Boyden).

\section{Ex vivo brain slice preparation}

Mice were anaesthetised using $4 \%$ isoflurane (Oxford University Veterinary Services) and were sacrificed by decapitation after the pedal reflex was abolished. Brains were extracted in warm (30 $35^{\circ} \mathrm{C}$ ) sucrose solution ( $34.5 \mathrm{mM} \mathrm{NaCl}, 3 \mathrm{mM} \mathrm{KCl}, 7.4 \mathrm{mM} \mathrm{MgSO}_{4} .7 \mathrm{H} 2 \mathrm{O}, 150 \mathrm{mM}$ sucrose, $1 \mathrm{mM}$ $\mathrm{CaCl}_{2}, 1.25 \mathrm{mM} \mathrm{NaH}_{2} \mathrm{PO}_{4}, 25 \mathrm{mM} \mathrm{NaHCO}_{3}$ and $15 \mathrm{mM}$ glucose) and transverse hippocampal slices 
of $350 \mu \mathrm{m}$ thickness were cut using a Leica vibratome (VT 1200S) (Huang et al. 2013). Slices were then immediately placed in an interface storing chamber containing warm (30-35 ${ }^{\circ} \mathrm{C}$ ) aCSF (126 $\mathrm{mM} \mathrm{NaCl}, 3.5 \mathrm{mM} \mathrm{KCl} 2 \mathrm{mM} \mathrm{MgSO}_{4}-7 \mathrm{H}_{2} \mathrm{O}, 1.25 \mathrm{mM} \mathrm{NaH}_{2} \mathrm{PO}_{4}, 24 \mathrm{mM} \mathrm{NaHCO}_{3}, 2 \mathrm{mM} \mathrm{CaCl}_{2}$ and 10 $\mathrm{mM}$ glucose) at least one hour to equilibrate. All solutions were bubbled with $95 \% \mathrm{O}_{2}$ and $5 \% \mathrm{CO}_{2}$ beginning 30 minutes before the procedure until the end of the experiment.

\section{Electrophysiology}

Extracellular recordings were conducted in an interface recording chamber at $33-34{ }^{\circ} \mathrm{C}$. Visualisation of the slices and electrode placement was performed using a Wild Heerbrugg dissection microscope. Local field potentials were recorded by inserting a borosilicate glass electrode filled with aCSF (tip resistance $=1-5 \mathrm{M} \Omega$ ) in CA3 pyramidal layer. Data were acquired and amplified (x 10) by Axoclamp 2A (Molecular Devices). The signal was further amplified x 100 and low pass filtered at $1 \mathrm{KHz}$ (LPBF48DG, NPI Electronic). The signal was then digitised at 5000 samples per second by a data acquisition board (ITC-16, InstruTECH) and recorded from the IgorPro (Wavemetrics). Gamma oscillations were induced by the application of $5 \mu \mathrm{M}$ carbachol (Cch). The LFP signal was quantified using real-time fast Fourier transform (FFT) analysis and oscillations were detected by a peak in the power spectrum at low -band frequencies $(25 \mathrm{~Hz}-49 \mathrm{~Hz}$ ). For unit recordings a linear 16 channel tungsten multielectrode array (MEA; MicroProbes) was lowered in the CA3 subfield. The array channels had 100 $\mu \mathrm{m}$ spacing to ensure full coverage of the hippocampus. The MEA was mounted on an RHD2132 Amplifier board and connected to the RHD2000 USB Interface Board (Intaan Technologies). Data were acquired at a rate of 20000 samples per second using the RHD2000 rhythm software (Intaan Technologies).

Intracellular recordings were always conducted in a single submerged chamber $\left(26-32{ }^{\circ} \mathrm{C}\right)$ using borosilicate glass pipettes (5-12 M). The signal was acquired through the MultiClamp 700B amplifier (Molecular Devices) and digitised at a rate of 10000 samples per second by a data acquisition board (ITC-18, InstruTECH) and was then recorded using the Igor Pro software. The signals were low pass filtered (Bessel) at $10 \mathrm{kHz}$ for current clamp mode and $3 \mathrm{kHz}$ for voltage clamp (VC) mode. Slice and cell visualisation were achieved using oblique illumination and monitored through a HAMATATSU ORCA - ER digital camera. Filtered white LED $(460+/-30 \mathrm{~nm}, 1.53 \mathrm{~mW}$, Thor Labs) via epiillumination was used to activate channelrhodopsin (ChR2). Filtered white LED (525 +/-20 nm, 1.45 $\mathrm{mW}$, Thor Labs) via epi-illumination was used to activate archaerhodopsin (Arch). For a power of 1.5 $\mathrm{mW}$, the illuminated area is $3.68 \mathrm{~mW} / \mathrm{mm}^{2}$. Cell attached recordings were performed in current 
clamp (IC) mode (Multiclamp software) using glass pipettes filled with aCSF. For whole cell current clamp recordings pipettes were filled with internal solution containing $110 \mathrm{mM}$ KGluconate, $40 \mathrm{mM}$ HEPES, 2 mM ATP-Mg, $0.3 \mathrm{mM} \mathrm{GTP-NaCl}, 4 \mathrm{mM} \mathrm{NaCl}$, (3-4 mg/ml biocytin, Sigma). For whole cell voltage recordings pipettes were filled with internal solution containing $140 \mathrm{mM}$ Cesium methanesulfonate, $5 \mathrm{mM} \mathrm{NaCl}, 10 \mathrm{mM}$ HEPES, 0.2 mM EGTA, 2 mM ATP-Mg, 3 mM GTP-Na, 5 mM QX-314, (3-4 $\mathrm{mg} / \mathrm{ml}$ biocytin). Series resistance compensation was not performed in all cells included for analysis. For perforated patch recordings the tip of the pipette was filled with a $\mathrm{KCl}-$ containing solution (150 mM KCl and 10mM HEPES, pH 7.2-7.3; Osmolality $300 \mathrm{mOsmol} / \mathrm{Kg}$ ). The rest of the pipette was filled with the same $\mathrm{KCl}$ solution containing $5 \mu \mathrm{M}$ gramicidin $\mathrm{D}$ (1:1000 DMSO dilution, Sigma) and $10 \mu \mathrm{M}$ Fluorescein (Sigma) to visualise if there was spontaneous rupture of the membrane during patching experiments.

\section{Light delivery}

For photo-activation (ChR2) experiments, light illumination was delivered through a fibre optic using a blue LED (470 +/- $20 \mathrm{~nm}$, Thorlabs, M470F3; max power at fibre optic tip = $10 \mathrm{~mW})$. For photoinhibition (Arch) experiments light illumination was delivered through a fibre optic by a green LED $(530+/-30 \mathrm{~nm}$, Thorlabs, M530F2; maximum power at fibre optic tip $=4.25 \mathrm{~mW})$ and with an amber LED (595 +/- 20nm, Doric, maximum power at fibre optic tip = $5 \mathrm{~mW})$. LED module output was controlled using the Igor Pro software. Laser photo-inhibition experiments were also performed with a green laser (MatchBox series, $532+/-0.5 \mathrm{~nm}$, maximum power at fibre optic that was used approx. $40 \mathrm{~mW}$ ). In these experiments the data were acquired at a rate of 10000 samples per second using IgorPro. The laser was operated manually, and the light duration was recorded using an Arduino Uno board that created a digital time stamp. Experiments were only included if the laser illumination duration was between 19.6 - 20.7 seconds. The area of light illumination was estimated to have a diameter of $1-2 \mathrm{~mm}$ and therefore for a power of $10 \mathrm{~mW}$ the light intensity was between $0.8-3.2 \mathrm{~mW} / \mathrm{mm}^{2}$.

\section{Histology and imaging}

After electrophysiological recordings, acute brain slices were fixed in $4 \%$ PFA overnight. Slices were kept in PBS (Phosphate Buffered Saline: $1.37 \mathrm{mM} \mathrm{NaCl}$, $2.7 \mathrm{mM} \mathrm{KCl}, 10 \mathrm{mM} \mathrm{Na}_{2} \mathrm{HPO}_{4}, 2 \mathrm{mM} \mathrm{KH}_{2} \mathrm{PO}_{4}$ ) at $4{ }^{\circ} \mathrm{C}$ for short-term storage. For biocytin labelling the slices were washed with $1 \mathrm{X}$ PBS 3-4 times and permeabilized with freshly prepared $0.3 \%$-Triton 1X PBS for 4 - 5 hours. Streptavidin conjugated 
to Alexa FluorTM 488 (Invitrogen S32355) in PBS-T $0.3 \%$ (1:500) was incubated overnight at $4{ }^{\circ} \mathrm{C}$. The slices were then washed 4 - 5 times in PBS for 2 hours. Slices were mounted on glass slides using mounting media (DAKO). Confocal images (1024 x 1024) were acquired on a Zeiss LSM700 upright confocal microscope using the $10 x$ air objective and digitally captured using the default LSM acquisition software. Pyramidal cell reconstruction was performed on neuron studio and simple neurite tracer plugin on Fiji.

\section{Analysis of local field potentials}

In order to characterise and analyse the oscillations, a hanning window was applied and the power spectra were calculated as the normalised magnitude square of the FFT (Igor Pro). The $50 \mathrm{~Hz}$ and $100 \mathrm{~Hz}$ frequencies were not included in the analysis to exclude the mains noise and its harmonic component. The oscillation amplitude was quantified firstly by measuring the peak of the power spectrum termed as peak power and secondly by measuring the area below the power spectrum plot in the gamma-band range $(20-100 \mathrm{~Hz})$ termed as power-area. The peak frequency of the oscillation was obtained by measuring the frequency at which the peak of the power spectrum occurred in the gamma-band range. In order to quantify when Cch-induced oscillations where abolished upon light stimulation and to exclude the peak frequencies of those oscillations from further analysis one of the two criteria had to be met. Firstly, an auto-correlation of the oscillations was computed and was fitted with a Gabor function $(f(x)=(A * \cos (2 \pi * f * x)) * e-$ $x 2 / 2 * t a u)$. The first criterion was met if the resulting Gabor fit had a linear correlation coefficient, $r>0.7$ and product of $\sqrt{f * \operatorname{tau}}>0.1$ ( $>0.15$ for frequencies higher than $50 \mathrm{~Hz}$ ). The second criterion was a power-area bigger than $125 \mu \mathrm{V}^{2}$ in the range of $+/-5 \mathrm{~Hz}$ of the peak frequency. The power-area was always included in the analysis even if oscillations were abolished. The power spectrum analysis for de novo oscillations was performed in the range of $52-149 \mathrm{~Hz}$ with the only criterion for oscillation presence being that the power-area in $+/-5 \mathrm{~Hz}$ of the peak frequency was larger than $40 \mu \mathrm{V}^{2}$. Hilbert transforms were used to obtain instantaneous gamma magnitude for sinusoidal modulation of gamma oscillations (band-pass filtered $20-120 \mathrm{~Hz}$ ). For visualisation purposes the magnitude of the continuous wavelet transform was used normalised by max value (Morlet wavelet; nondimensional frequency $=6$ ).

\section{Spike detection and analysis}

Unit detection was performed using custom-written procedures in MATLAB (Mathworks). Extracellular spikes from the 16 channel MEA were detected as described before by Quiroga and 
colleagues (Quiroga, Nadasdy \& Ben-Shaul, 2004; Quian Quiroga, 2009). Briefly, the MEA data were processed with an elliptical band-pass filter (for spike detection: 4th order, $300-3000 \mathrm{~Hz}$, for spike sorting: 2nd order, $300-6000 \mathrm{~Hz}$ ). Spikes were detected as signals exceeding 5 standard deviation (s.d.) of the noise $5 * \sigma n=$ median $\{|x| / 0.6745\}$. Signals that exceeded 10 times the s.d. of the detected spike amplitudes were eliminated as artefacts/population spikes. Subsequently, spikes that had peaks occurring at the same time $(<0.1 \mathrm{~ms})$ across channels were grouped together as one unit. This prevented detection of the same unit more than once. Clustering of the detected spikes was performed using custom-written procedures in Igor Pro. A spike sorting procedure adapted from Fee and colleagues was used to explore whether neurons displaying specific spike waveforms were selectively recruited by optogenetic stimulation (Fee, Mitra \& Kleinfeld, 1996). Briefly, spike metrics were converted into z scores, over-clustered using an in-built k-means algorithm, and progressively aggregated if the intercluster distance was $<2.5$ and merging did not produce more violations of refractory period of $2 \mathrm{~ms}$. Analysis was performed on the clustered spikes, with autocorrelation and cross-correlation plots used to validate the clustering procedure. Spike metrics from the average waveform for each cluster were used to identify different waveform types via a k-means algorithm. This clustering procedure is likely to be conservative, and underestimate the firing rate of individual neurons, but was deemed sufficiently robust to detect any bias in optogenetic recruitment. A single unit cluster was identified if it i) had less than $1.4 \%$ of its total spike waveforms within 2 ms of its refractory period and ii) consisted of more than 800 members. When a cluster did not obey these criteria, it was merged with other clusters that had similar action potential waveforms giving rise to a multi-unit cluster.

Clusters were identified as expressing ChR2 if the spike rate in the first $100 \mathrm{~ms}$ of the step stimulus was 3 s.d. above the baseline spike rate. The remaining clusters were classified based on the the delay between the negative and positive peaks in the average waveform as fast-spiking (<0.6 ms) or regular-spiking (>=0.6 ms). The Activation Index was calculated over the last second of the step stimulus as the difference between the light-induced and baseline spikes rates divided by their sum, and designed to measure sustained firing. The Theta Modulation Index was calculated as the rank correlation coefficient between the spike time histogram and the theta-modulated amplitude of the light stimulus.

\section{Statistics}

Repeated measures ANOVA (rmANOVA) was performed in SPSS with Greenhouse-Geisser correction where required (i.e. significance in Mauchly's test for sphericity) and followed by 
870 Bonferroni-corrected post-hoc paired t-tests. Linear correlations, circular correlations, and 871 Bonferroni-corrected one sample t-tests were performed using Igor Pro. Scatter-bar charts were 872 generated using PRISM. Circular statistics of spike phase relative to ongoing oscillations in the LFP 873 were calculated using in-built functions in Igor Pro. The measurements spiking rates deviated from 874 normality, and were analysed using non-parametric statistical tests performed in SPSS: differences 875 between cell types were analysed using Kruskal-Wallis Test, followed by posthoc Dunn's test, with 876 Bonferroni correction for multiple comparisons. Differences across stimulus types (step and theta) 877 were analysed using the Wilcoxon signed rank test, and the significance of modulation indices 878 analysed using the one-sample Wilcoxon signed rank test $\left(\mathrm{H}_{0}=0\right)$. In all figures, the bar charts display 879 the average value and the error bars represent the standard error of the mean, unless explicitly 880 stated otherwise. Stars represent significance values where $* p<0.05,{ }^{*} p<0.01$ and ${ }^{* * *} p<0.001$. 